\title{
CONNES-AMENABILITY OF BIDUAL AND WEIGHTED SEMIGROUP ALGEBRAS
}

\author{
MATTHEW DAWS
}

\begin{abstract}
We investigate the notion of Connes-amenability, introduced by Runde in [10], for bidual algebras and weighted semigroup algebras. We provide some simplifications to the notion of a $\sigma W C$-virtual diagonal, as introduced in [13], especially in the case of the bidual of an Arens regular Banach algebra. We apply these results to discrete, weighted, weakly cancellative semigroup algebras, showing that these behave in the same way as $\mathrm{C}^{*}$-algebras with regards Connes-amenability of the bidual algebra. We also show that for each one of these cancellative semigroup algebras $l^{1}(S, \omega)$, we have that $l^{1}(S, \omega)$ is Connes-amenable (with respect to the canonical predual $c_{0}(S)$ ) if and only if $l^{1}(S, \omega)$ is amenable, which is in turn equivalent to $S$ being an amenable group, and the weight satisfying a certain restrictive condition. This latter point was first shown by Grønbæk in [6], but we provide a unified proof. Finally, we consider the homological notion of injectivity, and show that here, weighted semigroup algebras do not behave like $\mathrm{C}^{*}$-algebras.
\end{abstract}

\section{Introduction}

We first fix some notation, following [2]. For a Banach space $E$, we let $E^{\prime}$ be its dual space, and for $\mu \in E^{\prime}$ and $x \in E$, we write $\langle\mu, x\rangle=\mu(x)$ for notational convenience. We then have the canonical map $\kappa_{E}: E \rightarrow E^{\prime \prime}$ defined by $\left\langle\kappa_{E}(x), \mu\right\rangle=\langle\mu, x\rangle$ for $\mu \in E^{\prime}, x \in E$. For Banach spaces $E$ and $F$, we write $\mathscr{B}(E, F)$ for the Banach space of bounded linear maps between $E$ and $F$; we write $\mathscr{B}(E, E)=\mathscr{B}(E)$; we write $T^{\prime}$ for the adjoint of $T \in \mathscr{B}(E, F)$. We use the notion of Banach left $\mathscr{A}$-modules, right modules and bimodules as in [2].

A linear map $d: \mathscr{A} \rightarrow E$ between a Banach algebra $\mathscr{A}$ and a Banach $\mathscr{A}$-bimodule $E$ is a derivation if $d(a b)=a \cdot d(b)+d(a) \cdot b$ for $a, b \in \mathscr{A}$. For $x \in E$, we define $\delta_{x}: \mathscr{A} \rightarrow E$ by $\delta_{x}(a)=a \cdot x-x \cdot a$. Then $\delta_{x}$ is a derivation, called an inner derivation.

A Banach algebra $\mathscr{A}$ is amenable if every derivation $d: \mathscr{A} \rightarrow E^{\prime}$ to a dual bimodule is inner. For example, a $\mathrm{C}^{*}$-algebra $\mathscr{A}$ is amenable if and only if $\mathscr{A}$ is nuclear; a group algebra $L^{1}(G)$ is amenable if and only if the locally compact group $G$ is amenable (which is the motivating example). See [14] for further discussions of amenability and related notions. 
Let $E$ be a Banach space and $F$ a closed subspace of $E$. Then we naturally, isometrically, identify $F^{\prime}$ with $E^{\prime} / F^{\circ}$, where

$$
F^{\circ}=\left\{\mu \in E^{\prime}:\langle\mu, x\rangle=0 \quad(x \in F)\right\} .
$$

Definition 1.1. Let $E$ be a Banach space and $E_{*}$ be a closed subspace of $E^{\prime}$. Let $\pi_{E_{*}}: E^{\prime \prime} \rightarrow E^{\prime \prime} / E_{*}^{\circ}$ be the quotient map, and suppose that $\pi_{E_{*}} \circ \kappa_{E}$ is an isomorphism from $E$ to $E_{*}^{\prime}$. Then we say that $E$ is a dual Banach space with predual $E_{*}$.

When $\mathscr{A}$ is a dual Banach space with predual $\mathscr{A}_{*}$ which is also a submodule of $\mathscr{A}^{\prime}$ we say that $\mathscr{A}$ is a dual Banach algebra.

For a dual Banach algebra $\mathscr{A}$ with predual $\mathscr{A}_{*}$, we henceforth identify $\mathscr{A}$ with $\mathscr{A}_{*}^{\prime}$. Thus we get a weak*-topology on $\mathscr{A}$, which we denote by $\sigma\left(\mathscr{A}, \mathscr{A}_{*}\right)$.

As noticed by Runde (see [10]), there are very few Banach algebras which are both dual and amenable. For von Neumann algebras, which are the motivating example of dual Banach algebras, there is a weaker notion of amenablity, called Connes-amenability, which has a natural generalisation to the case of dual Banach algebras.

Definition 1.2. Let $\mathscr{A}$ be a dual Banach algebra with predual $\mathscr{A}_{*}$. Let $E$ be a Banach $\mathscr{A}$-bimodule. Then $E^{\prime}$ is a $w^{*}$-Banach $\mathscr{A}$-bimodule if, for each $\mu \in E^{\prime}$, the maps

$$
\mathscr{A} \rightarrow E^{\prime}, \quad a \mapsto\left\{\begin{array}{l}
a \cdot \mu, \\
\mu \cdot a
\end{array}\right.
$$

are $\sigma\left(\mathscr{A}, \mathscr{A}_{*}\right)-\sigma\left(E^{\prime}, E\right)$ continuous.

Then $\left(\mathscr{A}, \mathscr{A}_{*}\right)$ is Connes-amenable if, for each $\mathrm{w}^{*}$-Banach $\mathscr{A}$-bimodule $E^{\prime}$, each derivation $d: \mathscr{A} \rightarrow E^{\prime}$, which is $\sigma\left(\mathscr{A}, \mathscr{A}_{*}\right)-\sigma\left(E^{\prime}, E\right)$ continuous, is inner.

Given a Banach algebra $\mathscr{A}$, we define bilinear maps $\mathscr{A}^{\prime \prime} \times \mathscr{A}^{\prime} \rightarrow \mathscr{A}^{\prime}$ and $\mathscr{A}^{\prime} \times \mathscr{A}^{\prime \prime} \rightarrow \mathscr{A}^{\prime}$ by

$\langle\Phi \cdot \mu, a\rangle=\langle\Phi, \mu \cdot a\rangle\langle\mu \cdot \Phi, a\rangle=\langle\Phi, a \cdot \mu\rangle \quad\left(\Phi \in \mathscr{A}^{\prime \prime}, \mu \in \mathscr{A}^{\prime}, a \in \mathscr{A}\right)$.

We then define two bilinear maps $\square, \diamond: \mathscr{A}^{\prime \prime} \times \mathscr{A}^{\prime \prime} \rightarrow \mathscr{A}^{\prime \prime}$ by

$$
\langle\Phi \square \Psi, \mu\rangle=\langle\Phi, \Psi \cdot \mu\rangle\langle\Phi \diamond \Psi, \mu\rangle=\langle\Psi, \mu \cdot \Phi\rangle \quad\left(\Phi, \Psi \in \mathscr{A}^{\prime \prime}, \mu \in \mathscr{A}^{\prime}\right) .
$$

We can check that $\square$ and $\diamond$ are actually algebra products, called the first and second Arens products respectively. Then $\kappa_{A}: \mathscr{A} \rightarrow \mathscr{A}^{\prime \prime}$ is a homomorphism with respect to either Arens product. When $\square=\diamond$, we say that $\mathscr{A}$ is Arens regular. In particular, when $\mathscr{A}$ is Arens regular, we may check that $\mathscr{A}^{\prime \prime}$ is a dual Banach algebra with predual $\mathscr{A}^{\prime}$. 
Theorem 1.3. Let $\mathscr{A}$ be an Arens regular Banach algebra. When $\mathscr{A}$ is amenable, $\mathscr{A}^{\prime \prime}$ is Connes-amenable. If $\kappa_{\mathscr{A}}(\mathscr{A})$ is an ideal in $\mathscr{A}^{\prime \prime}$ and $\mathscr{A}^{\prime \prime}$ is Connes-amenable, then $\mathscr{A}$ is amenable.

Let $\mathscr{A}$ be a $\mathrm{C}^{*}$-algebra. Then $\mathscr{A}$ is Arens regular, and $\mathscr{A}^{\prime \prime}$ is Connesamenable if and only if $\mathscr{A}$ is amenable.

Proof. The first statements are [10, Corollary 4.3] and [10, Theorem 4.4]. The statement about $\mathrm{C}^{*}$-algebras is detailed in [14, Chapter 6].

Another class of Connes-amenable dual Banach algebras is given by Runde in [11], where it is shown that $M(G)$, the measure algebra of a locally compact group $G$, is amenable if and only if $G$ is amenable.

The organisation of this paper is as follows. Firstly, we study intrinsic characterisations of amenability, recalling a result of Runde from [13]. We then simplify these conditions in the case of Arens regular Banach algebras. We recall the notion of an injective module, and quickly note how Connesamenability can be phrased in this language. The final section of the paper then applies these ideas to weighted semigroup algebras. We finish with some open questions.

\section{Characterisations of amenability}

Let $E$ and $F$ be Banach spaces, and form the algebraic tensor product $E \otimes F$. We can norm $E \otimes F$ with the projective tensor norm, defined as

$$
\|u\|_{\pi}=\inf \left\{\sum_{k=1}^{n}\left\|x_{k}\right\|\left\|y_{k}\right\|: u=\sum_{k=1}^{n} x_{k} \otimes y_{k}\right\} \quad(u \in E \otimes F) .
$$

Then the completion of $\left(E \otimes F,\|\cdot\|_{\pi}\right)$ is $E \widehat{\otimes} F$, the projective tensor product of $E$ and $F$.

Let $\mathscr{A}$ be a Banach algebra. Then $\mathscr{A} \widehat{\otimes} \mathscr{A}$ is a Banach $\mathscr{A}$-bimodule for the module actions given by

$a \cdot(b \otimes c)=a b \otimes c, \quad(b \otimes c) \cdot a=b \otimes c a \quad(a \in \mathscr{A}, b \otimes c \in \mathscr{A} \widehat{\otimes} \mathscr{A})$.

Define $\Delta_{\mathscr{A}}: \mathscr{A} \widehat{\otimes} \mathscr{A} \rightarrow \mathscr{A}$ by $\Delta_{\mathscr{A}}(a \otimes b)=a b$. Then $\Delta_{\mathscr{A}}$ is an $\mathscr{A}$-bimodule homomorphism. Let $M \in(\mathscr{A} \widehat{\otimes} \mathscr{A})^{\prime \prime}$ be such that

$$
a \cdot M=M \cdot a, \quad \Delta_{\mathscr{A}}^{\prime \prime}(M) \cdot a=\kappa_{\mathscr{A}}(a) \quad(a \in \mathscr{A}) .
$$

The $M$ is a virtual diagonal for $\mathscr{A}$. It is well-known that $\mathscr{A}$ is an amenable Banach algebra if and only if $\mathscr{A}$ has a virtual diagonal. 
Definition 2.1. Let $\mathscr{A}$ be a dual Banach algebra with predual $\mathscr{A}_{*}$, and let $E$ be a Banach $\mathscr{A}$-bimodule. Then $x \in \sigma W C(E)$ if and only if the maps $\mathscr{A} \rightarrow E$,

$$
a \mapsto\left\{\begin{array}{l}
a \cdot x, \\
x \cdot a
\end{array}\right.
$$

are $\sigma\left(\mathscr{A}, \mathscr{A}_{*}\right)-\sigma\left(E, E^{\prime}\right)$ continuous.

It is clear that $\sigma W C(E)$ is a closed submodule of $E$. The $\mathscr{A}$-bimodule homomorphism $\Delta_{\mathscr{A}}$ has adjoint $\Delta_{\mathscr{A}}^{\prime}: \mathscr{A}^{\prime} \rightarrow(\mathscr{A} \widehat{\otimes} \mathscr{A})^{\prime}$. In [13, Corollary 4.6] it is shown that $\Delta_{\mathscr{A}}^{\prime}\left(\mathscr{A}_{*}\right) \subseteq \sigma W C\left((\mathscr{A} \widehat{\otimes} \mathscr{A})^{\prime}\right)$. Consequently, we can view $\Delta_{\mathscr{A}}^{\prime}$ as a map $\mathscr{A}_{*} \rightarrow \sigma W C\left((\mathscr{A} \widehat{\otimes} \mathscr{A})^{\prime}\right)$, and hence view $\Delta_{\mathscr{A}}^{\prime \prime}$ as a map $\sigma W C((\mathscr{A} \widehat{\otimes}$ $\left.\mathscr{A})^{\prime}\right)^{\prime} \rightarrow \mathscr{A}_{*}^{\prime}=\mathscr{A}$, denoted by $\tilde{\Delta}_{\mathscr{A}}$. Let $M \in \sigma W C\left((\mathscr{A} \widehat{\otimes} \mathscr{A})^{\prime}\right)^{\prime}$ be such that

$$
a \cdot M=M \cdot a, \quad a \tilde{\Delta}_{\mathscr{A}}(M)=a \quad(a \in \mathscr{A}) .
$$

The $M$ is a $\sigma W C$-virtual diagonal for $\mathscr{A}$.

Theorem 2.2. Let $\mathscr{A}$ be a dual Banach algebra with predual $\mathscr{A}_{*}$. Then the following are equivalent:

(1) $\mathscr{A}$ is Connes-amenable;

(2) $\mathscr{A}$ has a $\sigma W C$-virtual diagonal.

Proof. This is [13, Theorem 4.8].

In particular, we see that a Connes-amenable Banach algebra is unital (which can of course be shown in an elementary fashion, as in [10, Proposition 4.1]).

\section{Connes-amenability for biduals of algebras}

Recall Gantmacher's theorem, which states that a bounded linear map $T$ : $E \rightarrow F$ between Banach spaces $E$ and $F$ is weakly compact if and only if $T^{\prime \prime}\left(E^{\prime \prime}\right) \subseteq \kappa_{F}(F)$. We write $\mathscr{W}(E, F)$ for the collection of weakly compact operators in $\mathscr{B}(E, F)$.

Lemma 3.1. Let $E$ be a dual Banach space with predual $E_{*}$, let $F$ be a Banach space, and let $T \in \mathscr{B}\left(E, F^{\prime}\right)$. Then the following are equivalent, and in particular each imply that $T$ is weakly compact:

(1) $T$ is $\sigma\left(E, E_{*}\right)-\sigma\left(F^{\prime}, F^{\prime \prime}\right)$ continuous;

(2) $T^{\prime}\left(F^{\prime \prime}\right) \subseteq \kappa_{E_{*}}\left(E_{*}\right)$;

(3) there exists $S \in \mathscr{W}\left(F, E_{*}\right)$ such that $S^{\prime}=T$.

Proof. That (1) and (2) are equivalent is standard. 
Suppose that (2) holds, so that we may define $S \in \mathscr{B}\left(F, E_{*}\right)$ by $\kappa_{E_{*}} \circ S=$ $T^{\prime} \circ \kappa_{F}$. Then, for $x \in E$ and $y \in F$, we have

$$
\langle x, S(y)\rangle=\left\langle T^{\prime}\left(\kappa_{F}(y)\right), x\right\rangle=\langle T(x), y\rangle,
$$

so that $S^{\prime}=T$. Then $S^{\prime \prime}\left(F^{\prime \prime}\right)=T^{\prime}\left(F^{\prime \prime}\right) \subseteq \kappa_{E_{*}}\left(E_{*}\right)$, so that $S$ is weakly compact, by Gantmacher's Theorem, so that (3) holds.

Conversely, if (3) holds, as $S$ is weakly compact, we have $\kappa_{E *}\left(E_{*}\right) \supseteq$ $S^{\prime \prime}\left(F^{\prime \prime}\right)=T^{\prime}\left(F^{\prime \prime}\right)$, so that $(2)$ holds.

It is standard that for Banach spaces $E$ and $F$, we have $(E \widehat{\otimes} F)^{\prime}=\mathscr{B}\left(F, E^{\prime}\right)$ with duality defined by

$$
\langle T, x \otimes y\rangle=\langle T(y), x\rangle \quad\left(T \in \mathscr{B}\left(F, E^{\prime}\right), x \otimes y \in E \widehat{\otimes} F\right) .
$$

Then we see, for $a, b, c \in \mathscr{A}$ and $T \in(\mathscr{A} \widehat{\otimes} \mathscr{A})^{\prime}=\mathscr{B}\left(\mathscr{A}, \mathscr{A}^{\prime}\right)$, that $\langle a$. $T, b \otimes c\rangle=\langle T(c a), b\rangle$ and that $\langle T \cdot a, b \otimes c\rangle=\langle T(c), a b\rangle=\langle T(c) \cdot a, b\rangle$ so that

$$
(a \cdot T)(c)=T(c a), \quad(T \cdot a)(c)=T(c) \cdot a \quad\left(a, c \in \mathscr{A}, T: \mathscr{A} \rightarrow \mathscr{A}^{\prime}\right) .
$$

Notice that we could also have defined $(E \widehat{\otimes} F)^{\prime}$ to be $\mathscr{B}\left(E, F^{\prime}\right)$. This would induce a different bimodule structure on $\mathscr{B}\left(\mathscr{A}, \mathscr{A}^{\prime}\right)$, but we shall see in Section 4 that our chosen convention seems more natural for the task at hand.

Proposition 3.2. Let $\mathscr{A}$ be a dual Banach algebra with predual $\mathscr{A}_{*}$. For $T \in \mathscr{B}\left(\mathscr{A}, \mathscr{A}^{\prime}\right)=(\mathscr{A} \widehat{\otimes} \mathscr{A})^{\prime}$, define maps $\phi_{r}, \phi_{l}: \mathscr{A} \widehat{\otimes} \mathscr{A} \rightarrow \mathscr{A}^{\prime}$ by

$$
\phi_{r}(a \otimes b)=T^{\prime} \kappa_{\mathscr{A}}(a) \cdot b, \quad \phi_{l}(a \otimes b)=a \cdot T(b) \quad(a \otimes b \in \mathscr{A} \widehat{\otimes} \mathscr{A}) .
$$

Then $T \in \sigma W C\left(\mathscr{B}\left(\mathscr{A}, \mathscr{A}^{\prime}\right)\right)$ if and only if $\phi_{r}$ and $\phi_{l}$ are weakly compact and have ranges contained in $\kappa_{\mathscr{A}_{*}}\left(\mathscr{A}_{*}\right)$.

Proof. For $T \in \mathscr{B}\left(\mathscr{A}, \mathscr{A}^{\prime}\right)=(\mathscr{A} \widehat{\otimes} \mathscr{A})^{\prime}$, define $R_{T}, L_{T}: \mathscr{A} \rightarrow(\mathscr{A} \widehat{\otimes}$ $\mathscr{A})^{\prime}$ by $R_{T}(a)=a \cdot T$ and $L_{T}=T \cdot a$, for $a \in \mathscr{A}$. By definition, $T \in$ $\sigma W C\left(\mathscr{B}\left(\mathscr{A}, \mathscr{A}^{\prime}\right)\right)$ if and only if $R_{T}$ and $L_{T}$ are $\sigma\left(\mathscr{A}, \mathscr{A}_{*}\right)-\sigma\left(\mathscr{B}\left(\mathscr{A}, \mathscr{A}^{\prime}\right)\right.$, $\left.(\mathscr{A} \widehat{\otimes} \mathscr{A})^{\prime \prime}\right)$ continuous. By Lemma 3.1, this is if and only if there exist $\varphi_{r}, \varphi_{l} \in$ $\mathscr{W}\left(\mathscr{A} \widehat{\otimes} \mathscr{A}, \mathscr{A}_{*}\right)$ such that $\varphi_{r}^{\prime}=R_{T}$ and $\varphi_{l}^{\prime}=L_{T}$.

For $a \otimes b \in \mathscr{A} \widehat{\otimes} \mathscr{A}$ and $c \in \mathscr{A}$, we see that

$$
\begin{aligned}
\left\langle c, \varphi_{r}(a \otimes b)\right\rangle & =\left\langle R_{T}(c), a \otimes b\right\rangle=\langle c \cdot T, a \otimes b\rangle=\langle T(b c), a\rangle \\
& =\left\langle T^{\prime} \kappa_{\mathscr{A}}(a), b c\right\rangle=\left\langle T^{\prime} \kappa_{\mathscr{A}}(a) \cdot b, c\right\rangle=\left\langle\phi_{r}(a \otimes b), c\right\rangle, \\
\left\langle c, \varphi_{l}(a \otimes b)\right\rangle & =\left\langle L_{T}(c), a \otimes b\right\rangle=\langle T \cdot c, a \otimes b\rangle=\langle T(b), c a\rangle \\
& =\langle a \cdot T(b), c\rangle=\left\langle\phi_{l}(a \otimes b), c\right\rangle .
\end{aligned}
$$


Thus $\kappa_{\mathscr{A}_{*}} \circ \varphi_{r}=\phi_{r}$ and $\kappa_{\mathscr{A}_{*}} \circ \varphi_{l}=\phi_{l}$. Consequently, we see that $T \in$ $\sigma W C\left(\mathscr{B}\left(\mathscr{A}, \mathscr{A}^{\prime}\right)\right)$ if and only if $\phi_{r}$ and $\phi_{l}$ are weakly compact and take values in $\kappa_{\mathscr{A}_{*}}\left(\mathscr{A}_{*}\right)$.

The following definition is [13, Definition 4.1].

Definition 3.3. Let $\mathscr{A}$ be a Banach algebra and let $E$ be a Banach $\mathscr{A}$ bimodule. An element $x \in E$ is weakly almost periodic if the maps

$$
\mathscr{A} \rightarrow E, \quad a \mapsto\left\{\begin{array}{l}
a \cdot x, \\
x \cdot a
\end{array}\right.
$$

are weakly compact. The collection of weakly almost periodic elements in $E$ is denoted by $\operatorname{WAP}(E)$.

Lemma 3.4. Let $\mathscr{A}$ be a Banach algebra, and let $T \in \mathscr{B}\left(\mathscr{A}, \mathscr{A}^{\prime}\right)=$ $(\mathscr{A} \widehat{\otimes} \mathscr{A})^{\prime}$. Let $\phi_{r}, \phi_{l}: \mathscr{A} \widehat{\otimes} \mathscr{A} \rightarrow \mathscr{A}^{\prime}$ be as above. Then $T \in \operatorname{WAP}\left(\mathscr{B}\left(\mathscr{A}, \mathscr{A}^{\prime}\right)\right)$ if and only if $\phi_{r}$ and $\phi_{l}$ are weakly compact.

Proof. Let $R_{T}, L_{T}: \mathscr{A} \rightarrow \mathscr{B}\left(\mathscr{A}, \mathscr{A}^{\prime}\right)$ be as in the above proof. By definition, $T \in \operatorname{WAP}\left(\mathscr{B}\left(\mathscr{A}, \mathscr{A}^{\prime}\right)\right)$ if and only if $L_{T}$ and $R_{T}$ are weakly compact. We can verify that

$$
\phi_{r}^{\prime} \circ \kappa_{\mathscr{A}}=R_{T}, \quad \phi_{l}^{\prime} \circ \kappa_{\mathscr{A}}=L_{T}, \quad R_{T}^{\prime} \circ \kappa_{\mathscr{A} \hat{\otimes} \mathscr{A}}=\phi_{r}, \quad L_{T}^{\prime} \circ \kappa_{\mathscr{A}} \widehat{\otimes} \mathscr{A}=\phi_{l},
$$

which completes the proof.

Corollary 3.5. Let $\mathscr{A}$ be a unital, dual Banach algebra with predual $\mathscr{A}_{*}$, and let $T \in \mathscr{B}\left(\mathscr{A}, \mathscr{A}^{\prime}\right)=(\mathscr{A} \widehat{\otimes} \mathscr{A})^{\prime}$. The following are equivalent, and, in particular, each imply that $T$ is weakly compact:

(1) $T \in \sigma W C\left(\mathscr{B}\left(\mathscr{A}, \mathscr{A}^{\prime}\right)\right)$;

(2) $T(\mathscr{A}) \subseteq \kappa_{\mathscr{A}_{*}}\left(\mathscr{A}_{*}\right), T^{\prime}\left(\kappa_{\mathscr{A}}(\mathscr{A})\right) \subseteq \kappa_{\mathscr{A}_{*}}\left(\mathscr{A}_{*}\right)$, and $T \in \sigma W C\left(\mathscr{B}\left(\mathscr{A}, \mathscr{A}^{\prime}\right)\right)$;

(3) $T(\mathscr{A}) \subseteq \kappa_{\mathscr{A}_{*}}\left(\mathscr{A}_{*}\right), T^{\prime}\left(\kappa_{\mathscr{A}}(\mathscr{A})\right) \subseteq \kappa_{\mathscr{A}_{*}}\left(\mathscr{A}_{*}\right)$, and $T \in \operatorname{WAP}\left(\mathscr{B}\left(\mathscr{A}, \mathscr{A}^{\prime}\right)\right)$.

Proof. Let $e_{\mathscr{A}}$ be the unit of $\mathscr{A}$, so that for $a \in \mathscr{A}$, we have $T(a)=$ $\phi_{l}\left(e_{\mathscr{A}} \otimes a\right)$ and $T^{\prime} \kappa_{\mathscr{A}}(a)=\phi_{r}\left(a \otimes e_{\mathscr{A}}\right)$, which shows that (1) implies (2); clearly (2) implies (1).

As $\mathscr{A}_{*}$ is an $\mathscr{A}$-bimodule, (2) and (3) are equivalent by an application of Lemma 3.4 and Proposition 3.2.

Theorem 3.6. Let $\mathscr{A}$ be a dual Banach algebra with predual $\mathscr{A}_{*}$. Then $\mathscr{A}$ is Connes-amenable if and only if $\mathscr{A}$ is unital and there exists $M \in(\mathscr{A} \widehat{\otimes} \mathscr{A})^{\prime \prime}$ such that:

(1) $\langle M, a \cdot T-T \cdot a\rangle=0$ for $a \in \mathscr{A}$ and $T \in \sigma W C\left(\mathscr{W}\left(\mathscr{A}, \mathscr{A}^{\prime}\right)\right)$;

(2) $\kappa_{\mathscr{A}_{*}}^{\prime} \Delta_{\mathscr{A}}^{\prime \prime}(M)=e_{\mathscr{A}}$, where $e_{\mathscr{A}}$ is the unit of $\mathscr{A}$. 
Proof. As $\sigma W C\left((\mathscr{A} \widehat{\otimes} \mathscr{A})^{\prime}\right)^{\prime}$ is a quotient of $(\mathscr{A} \widehat{\otimes} \mathscr{A})^{\prime \prime}$, this is just a re-statement of Theorem 2.2 .

When $\mathscr{A}$ is an Arens regular Banach algebra, $\mathscr{A}^{\prime \prime}$ is a dual Banach algebra with canonical predual $\mathscr{A}^{\prime}$. In this case, we can make some significant simplifications in the characterisation of when $\mathscr{A}^{\prime \prime}$ is Connes-amenable.

For a Banach algebra $\mathscr{A}$, we define the map $\kappa_{\mathscr{A}} \otimes \kappa_{\mathscr{A}}: \mathscr{A} \widehat{\otimes} \mathscr{A} \rightarrow \mathscr{A}^{\prime \prime} \widehat{\otimes} \mathscr{A}^{\prime \prime}$ by

$$
\left(\kappa_{\mathscr{A}} \otimes \kappa_{\mathscr{A}}\right)(a \otimes b)=\kappa_{\mathscr{A}}(a) \otimes \kappa_{\mathscr{A}}(b) \quad(a \otimes b \in \mathscr{A} \widehat{\otimes} \mathscr{A}) .
$$

We turn $\mathscr{A}^{\prime \prime} \widehat{\otimes} \mathscr{A}^{\prime \prime}$ into a Banach $\mathscr{A}$-bimodule in the canonical way. Then $\kappa_{\mathscr{A}} \otimes$ $\kappa_{\mathscr{A}}$ is an $\mathscr{A}$-bimodule homomorphism. The following is a simple verification.

Lemma 3.7. Let $\mathscr{A}$ be a Banach algebra. The map

$$
\iota_{\mathscr{A}}: \mathscr{B}\left(\mathscr{A}, \mathscr{A}^{\prime}\right) \rightarrow \mathscr{B}\left(\mathscr{A}^{\prime \prime}, \mathscr{A}^{\prime \prime \prime}\right) ; T \mapsto T^{\prime \prime}
$$

is an $\mathscr{A}$-bimodule homomorphism which is an isometry onto its range. Fur-

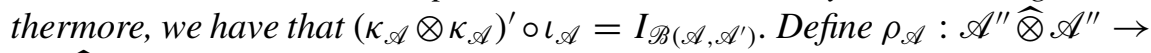
$(\mathscr{A} \widehat{\otimes} \mathscr{A})^{\prime \prime}$ by

$$
\left\langle\rho_{\mathscr{A}}(\tau), T\right\rangle=\left\langle T^{\prime \prime}, \tau\right\rangle \quad\left(\tau \in \mathscr{A}^{\prime \prime} \widehat{\otimes} \mathscr{A}^{\prime \prime}, T \in \mathscr{B}\left(\mathscr{A}, \mathscr{A}^{\prime}\right)=(\mathscr{A} \widehat{\otimes} \mathscr{A})^{\prime}\right) .
$$

Then $\rho_{\mathscr{A}}$ is a norm-decreasing $\mathscr{A}$-bimodule homomorphism which satisfies $\rho_{\mathscr{A}} \circ\left(\kappa_{\mathscr{A}} \otimes \kappa_{\mathscr{A}}\right)=\kappa_{\mathscr{A}} \widehat{\otimes} \mathscr{A}$.

For a Banach algebra $\mathscr{A}$, it is clear that $\mathscr{W}\left(\mathscr{A}, \mathscr{A}^{\prime}\right)$ is a sub- $\mathscr{A}$-bimodule of $\mathscr{B}\left(\mathscr{A}, \mathscr{A}^{\prime}\right)=(\mathscr{A} \widehat{\otimes} \mathscr{A})^{\prime}$.

Theorem 3.8. Let $\mathscr{A}$ be an Arens regular Banach algebra such that $\mathscr{A}^{\prime \prime}$ is unital, and let $T \in \mathscr{B}\left(\mathscr{A}^{\prime \prime}, \mathscr{A}^{\prime \prime \prime}\right)=\left(\mathscr{A}^{\prime \prime} \widehat{\otimes} \mathscr{A}^{\prime \prime}\right)^{\prime}$. Then the following are equivalent:

(1) $T \in \sigma W C\left(\mathscr{B}\left(\mathscr{A}^{\prime \prime}, \mathscr{A}^{\prime \prime \prime}\right)\right)$, where we treat $\mathscr{B}\left(\mathscr{A}^{\prime \prime}, \mathscr{A}^{\prime \prime \prime}\right)$ as an $\mathscr{A}^{\prime \prime}$ bimodule;

(2) $T=S^{\prime \prime}$ for some $S \in \operatorname{WAP}\left(\mathscr{W}\left(\mathscr{A}, \mathscr{A}^{\prime}\right)\right)$, where now we treat $\mathscr{W}\left(\mathscr{A}, \mathscr{A}^{\prime}\right)$ as an $\mathscr{A}$-bimodule.

Proof. We apply Corollary 3.5 to $\mathscr{A}^{\prime \prime}$, so that (1) is equivalent to $T$ being weakly compact, $T\left(\mathscr{A}^{\prime \prime}\right) \subseteq \kappa_{\mathscr{A}}\left(\mathscr{A}^{\prime}\right), T^{\prime}\left(\kappa_{\mathscr{A}^{\prime \prime}}\left(\mathscr{A}^{\prime \prime}\right)\right) \subseteq \kappa_{\mathscr{A}}\left(\mathscr{A}^{\prime}\right)$, and $T \in$ WAP $\left(\mathscr{B}\left(\mathscr{A}^{\prime \prime}, \mathscr{A}^{\prime \prime \prime}\right)\right)$. Thus, if (1) holds, then there exists $T_{0} \in \mathscr{W}\left(\mathscr{A}^{\prime \prime}, \mathscr{A}^{\prime}\right)$ such that $T=\kappa_{\mathscr{A}} \circ T_{0}$, and there exists $T_{1} \in \mathscr{W}\left(\mathscr{A}^{\prime \prime}, \mathscr{A}^{\prime}\right)$ such that $T^{\prime} \circ \kappa_{\mathscr{A}^{\prime \prime}}=$ $\kappa_{\mathscr{A}} \circ T_{1}$. Let $S=T_{0} \circ \kappa_{\mathscr{A}} \in \mathscr{W}\left(\mathscr{A}, \mathscr{A}^{\prime}\right)$. As before, we can check that $S^{\prime}=T_{1}$ and that $S^{\prime \prime}=T$. We know that the maps $L_{T}, R_{T}: \mathscr{A}^{\prime \prime} \rightarrow \mathscr{B}\left(\mathscr{A}^{\prime \prime}, \mathscr{A}^{\prime \prime \prime}\right)$, 
defined by $L_{T}(\Phi)=T \cdot \Phi$ and $R_{T}(\Phi)=\Phi \cdot T$ for $\Phi \in \mathscr{A}^{\prime \prime}$, are weakly compact. Define $L_{S}, R_{S}: \mathscr{A} \rightarrow \mathscr{B}\left(\mathscr{A}, \mathscr{A}^{\prime}\right)$ is an analogous manner, using $S \in \mathscr{W}\left(\mathscr{A}, \mathscr{A}^{\prime}\right)$. For $a \in \mathscr{A}, S \cdot a \in \mathscr{W}\left(\mathscr{A}, \mathscr{A}^{\prime}\right)$, so for $\Psi \in \mathscr{A}^{\prime \prime}$ and $b \in \mathscr{A}$,

$$
\begin{aligned}
\left\langle(S \cdot a)^{\prime}(\Psi), b\right\rangle & =\langle\Psi,(S \cdot a)(b)\rangle=\langle\Psi, S(b) \cdot a\rangle \\
& =\langle a \cdot \Psi, S(b)\rangle=\left\langle S^{\prime}(a \cdot \Psi), b\right\rangle .
\end{aligned}
$$

Thus, for $a \in \mathscr{A}$ and $\Phi, \Psi \in \mathscr{A}^{\prime \prime}$, we have that

$$
\left\langle\iota_{\mathscr{A}}\left(L_{S}(a)\right)(\Phi), \Psi\right\rangle=\left\langle(S \cdot a)^{\prime \prime}(\Phi), \Psi\right\rangle=\left\langle\Phi, S^{\prime}(a \cdot \Psi)\right\rangle=\left\langle S^{\prime \prime}(\Phi) \cdot a, \Psi\right\rangle,
$$

so that $\iota_{\mathscr{A}}\left(L_{S}(a)\right)(\Phi)=S^{\prime \prime}(\Phi) \cdot a$, and hence that $\iota_{\mathscr{A}}\left(L_{S}(a)\right)=S^{\prime \prime} \cdot a=T \cdot a=$ $T \cdot \kappa_{\mathscr{A}}(a)=L_{T}\left(\kappa_{\mathscr{A}}(a)\right)$. Thus we have that $L_{S}=\left(\kappa_{\mathscr{A}} \otimes \kappa_{\mathscr{A}}\right)^{\prime} \circ R_{T} \circ \kappa_{\mathscr{A}}$, so that $L_{S}$ is weakly compact. A similar calculation shows that $R_{S}$ is also weakly compact, so that $S \in \operatorname{WAP}\left(\mathscr{W}\left(\mathscr{A}, \mathscr{A}^{\prime}\right)\right)$. This shows that (1) implies (2).

Conversely, if (2) holds, then $L_{S}$ and $R_{S}$ are weakly compact. As $S$ is weakly compact, $T\left(\mathscr{A}^{\prime \prime}\right)=S^{\prime \prime}\left(\mathscr{A}^{\prime \prime}\right) \subseteq \kappa_{\mathscr{A}}\left(\mathscr{A}^{\prime}\right)$ and $T^{\prime}\left(\kappa_{\mathscr{A}} \mathscr{A}^{\prime \prime}\left(\mathscr{A}^{\prime \prime}\right)\right)=S^{\prime \prime \prime}\left(\kappa_{\mathscr{A}}\left(\mathscr{A}^{\prime \prime}\right)\right)$ $=\kappa_{\mathscr{A}}\left(S^{\prime}\left(\mathscr{A}^{\prime \prime}\right)\right) \subseteq \kappa_{\mathscr{A}}\left(\mathscr{A}^{\prime}\right)$, and $T$ is weakly compact. Thus, to show (1), we are required to show that $L_{T}$ and $R_{T}$ are weakly compact.

For $a, b \in \mathscr{A}$ and $\Phi \in \mathscr{A}^{\prime}$, we have

$$
\left\langle(a \cdot S)^{\prime}(\Phi), b\right\rangle=\langle\Phi, S(b a)\rangle=\left\langle a \cdot S^{\prime}(\Phi), b\right\rangle .
$$

Then, for $\Phi, \Psi \in \mathscr{A}^{\prime \prime}$ and $a \in \mathscr{A}$, we thus have

$$
\begin{aligned}
\left\langle R_{S}^{\prime}\left(\rho_{\mathscr{A}}(\Phi \otimes \Psi)\right), a\right\rangle & =\left\langle(a \cdot S)^{\prime \prime}, \Phi \otimes \Psi\right\rangle=\left\langle(a \cdot S)^{\prime \prime}(\Psi), \Phi\right\rangle \\
& =\left\langle\Psi, a \cdot S^{\prime}(\Phi)\right\rangle=\left\langle\Psi \cdot a, S^{\prime}(\Phi)\right\rangle \\
& =\left\langle\Psi \square \kappa_{\mathscr{A}}(a), S^{\prime}(\Phi)\right\rangle=\left\langle S^{\prime}(\Phi) \cdot \Psi, a\right\rangle .
\end{aligned}
$$

Hence we see that $R_{S}^{\prime}\left(\rho_{\mathscr{A}}(\Phi \otimes \Psi)\right)=S^{\prime}(\Phi) \cdot \Psi$. Let $U=R_{S}^{\prime} \circ \rho_{\mathscr{A}}: \mathscr{A}^{\prime \prime} \widehat{\otimes}$ $\mathscr{A}^{\prime \prime} \rightarrow \mathscr{A}^{\prime}$, so that as $R_{S}$ is weakly compact, so is $U$. Then, for $\Phi, \Psi, \Gamma \in \mathscr{A}^{\prime \prime}$, we have that

$$
\begin{aligned}
\left\langle U^{\prime}(\Gamma), \Phi \otimes \Psi\right\rangle & =\left\langle\Gamma, S^{\prime}(\Phi) \cdot \Psi\right\rangle=\left\langle\Psi \diamond \Gamma, S^{\prime}(\Phi)\right\rangle \\
& =\left\langle S^{\prime \prime}(\Psi \square \Gamma), \Phi\right\rangle=\left\langle\left(\Gamma \cdot S^{\prime \prime}\right)(\Psi), \Phi\right\rangle,
\end{aligned}
$$

so that $U^{\prime}(\Gamma)=\Gamma \cdot T$, that is, $U^{\prime}=R_{T}$, so that $R_{T}$ is weakly compact. Similarly, we can show that $L_{T}$ is weakly compact, completing the proof.

TheORem 3.9. Let $\mathscr{A}$ be an Arens regular Banach algebra. Then $\mathscr{A}^{\prime \prime}$ is Connes-amenable if and only if $\mathscr{A l}^{\prime \prime}$ is unital and there exists $M \in(\mathscr{A} \widehat{\otimes} \mathscr{A})^{\prime \prime}$ such that: 
(1) $\Delta_{\mathscr{A}}^{\prime \prime}(M)=e_{\mathscr{A}}$, the unit of $\mathscr{A}^{\prime \prime}$;

(2) $\langle M, a \cdot T-T \cdot a\rangle=0$ for each $a \in \mathscr{A}$ and each $T \in \operatorname{WAP}\left(\mathscr{W}\left(\mathscr{A}, \mathscr{A}^{\prime}\right)\right)$.

Proof. By Theorem 3.6, we wish to show that the existence of such an $M$ is equivalent to the existence of $N \in\left(\mathscr{A}^{\prime \prime} \widehat{\otimes} \mathscr{A}^{\prime \prime}\right)^{\prime \prime}$ such that:

(N1) $\kappa_{\mathscr{A}^{\prime}}^{\prime} \Delta_{\mathscr{A}^{\prime \prime}}^{\prime \prime}(N)=e_{\mathscr{A}^{\prime \prime}}$;

(N2) $\langle N, \Phi \cdot S-S \cdot \Phi\rangle=0$ for each $\Phi \in \mathscr{A}^{\prime \prime}$ and each

$S \in \sigma W C\left(\mathscr{B}\left(\mathscr{A}^{\prime \prime}, \mathscr{A}^{\prime \prime \prime}\right)\right)$.

We can verify that $\iota_{\mathscr{A}} \circ \Delta_{\mathscr{A}}^{\prime}=\Delta_{\mathscr{A}^{\prime \prime}}^{\prime} \circ \kappa_{\mathscr{A}}^{\prime}$, so that (N1) is equivalent to $\Delta_{\mathscr{A}}^{\prime \prime} l_{\mathscr{A}}^{\prime}(N)=e_{\mathscr{A}}$. For $S \in \sigma W C\left(\mathscr{B}\left(\mathscr{A}^{\prime \prime}, \mathscr{A}^{\prime \prime \prime}\right)\right)$, we know that $S=T^{\prime \prime}$ for some $T \in \operatorname{WAP}\left(\mathscr{W}\left(\mathscr{A}, \mathscr{A}^{\prime}\right)\right)$, by Theorem 3.8. That is, the maps $\phi_{r}$ and $\phi_{l}$, formed using $T$ as in Proposition 3.2, are weakly compact. Then, for $\Phi \in \mathscr{A}^{\prime \prime}$, $\phi_{r}^{\prime}(\Phi), \phi_{l}^{\prime}(\Phi) \in \mathscr{B}\left(\mathscr{A}, \mathscr{A}^{\prime}\right)$, and we can check that

$$
\phi_{r}^{\prime}(\Phi)(a)=\kappa_{\mathscr{A}}^{\prime} T^{\prime \prime}(a \cdot \Phi), \quad \phi_{l}^{\prime}(\Phi)(a)=T(a) \cdot \Phi \quad(a \in \mathscr{A}) .
$$

Then $\phi_{r}^{\prime}(\Phi)^{\prime}, \phi_{l}^{\prime}(\Phi)^{\prime} \in \mathscr{B}\left(\mathscr{A}^{\prime \prime}, \mathscr{A}^{\prime}\right)$ are the maps

$$
\phi_{r}^{\prime}(\Phi)^{\prime}(\Psi)=\Phi \cdot T^{\prime}(\Psi), \quad \phi_{l}^{\prime}(\Phi)^{\prime}(\Psi)=T^{\prime}(\Phi \square \Psi) \quad\left(\Psi \in \mathscr{A}^{\prime \prime}\right),
$$

where we remember that $T^{\prime \prime}\left(\mathscr{A}^{\prime \prime}\right) \subseteq \kappa_{\mathscr{A}}\left(\mathscr{A}^{\prime}\right)$. Consequently $\phi_{r}^{\prime}(\Phi)^{\prime \prime}, \phi_{l}^{\prime}(\Phi)^{\prime \prime}$ $\in \mathscr{B}\left(\mathscr{A}^{\prime \prime}, \mathscr{A}^{\prime \prime \prime}\right)$ are given by

$$
\phi_{r}^{\prime}(\Phi)^{\prime \prime}(\Psi)=T^{\prime \prime}(\Psi \square \Phi), \quad \phi_{l}^{\prime}(\Phi)^{\prime \prime}(\Psi)=T^{\prime \prime}(\Psi) \cdot \Phi \quad\left(\Psi \in \mathscr{A}^{\prime \prime}\right),
$$

where $\mathscr{A}^{\prime \prime \prime}$ is an $\mathscr{A}^{\prime \prime}$-bimodule, as $\mathscr{A}^{\prime \prime}$ is Arens regular. That is, $\phi_{r}^{\prime}(\Phi)^{\prime \prime}=\Phi \cdot S$ and $\phi_{l}^{\prime}(\Phi)^{\prime \prime}=S \cdot \Phi$. Hence (N2) is equivalent to

$$
\begin{aligned}
0=\left\langle N, \phi_{r}^{\prime}(\Phi)^{\prime \prime}-\phi_{l}^{\prime}(\Phi)^{\prime \prime}\right\rangle & =\left\langle N, \iota_{\mathscr{A}}\left(\phi_{r}^{\prime}(\Phi)-\phi_{l}^{\prime}(\Phi)\right)\right\rangle \\
& =\left\langle\iota_{\mathscr{A}}^{\prime}(N), \phi_{r}^{\prime}(\Phi)-\phi_{l}^{\prime}(\Phi)\right\rangle,
\end{aligned}
$$

for each $\Phi \in \mathscr{A}^{\prime \prime}$ and $S \in \sigma W C\left(\mathscr{B}\left(\mathscr{A}^{\prime \prime}, \mathscr{A}^{\prime \prime \prime}\right)\right)$. That is, (N2) is equivalent to

$$
\phi_{r}^{\prime \prime} \iota_{\mathscr{A}}^{\prime}(N)-\phi_{l}^{\prime \prime} \iota_{\mathscr{A}}^{\prime}(N)=0 \quad\left(S \in \sigma W C\left(\mathscr{B}\left(\mathscr{A}^{\prime \prime}, \mathscr{A}^{\prime \prime \prime}\right)\right)\right) .
$$

As $\phi_{r}$ and $\phi_{l}$ are weakly compact, $\phi_{r}^{\prime \prime}$ and $\phi_{l}^{\prime \prime}$ take values in $\kappa_{\mathscr{A}^{\prime}}\left(\mathscr{A}^{\prime}\right)$, and so (N2) is equivalent to

$$
0=\left\langle\phi_{r}^{\prime \prime} \iota_{\mathscr{A}}^{\prime}(N)-\phi_{l}^{\prime \prime} \iota_{\mathscr{A}}^{\prime}(N), \kappa_{\mathscr{A}}(a)\right\rangle=\left\langle\iota_{\mathscr{A}}^{\prime}(N), \phi_{r}^{\prime}\left(\kappa_{\mathscr{A}}(a)\right)-\phi_{l}^{\prime}\left(\kappa_{\mathscr{A}}(a)\right)\right\rangle,
$$

for each $a \in \mathscr{A}$ and each $S \in \sigma W C\left(\mathscr{B}\left(\mathscr{A}^{\prime \prime}, \mathscr{A}^{\prime \prime \prime}\right)\right)$. However, $\phi_{r}^{\prime}\left(\kappa_{\mathscr{A}}(a)\right)-$ $\phi_{l}^{\prime}\left(\kappa_{\mathscr{A}}(a)\right)=a \cdot T-T \cdot a$, so that (N2) is equivalent to

$$
0=\left\langle\iota_{\mathscr{A}}^{\prime}(N), a \cdot T-T \cdot a\right\rangle \quad(a \in \mathscr{A}),
$$


for each $T \in \mathscr{W}\left(\mathscr{A}, \mathscr{A}^{\prime}\right)$ such that $\phi_{r}$ and $\phi_{l}$ are weakly compact.

Thus we have established that (N1) holds for $N$ if and only if (1) holds for $M=\iota_{\mathscr{A}}^{\prime}(N)$, and that (N2) holds for $N$ if and only if (2) holds for $M=\iota_{\mathscr{A}}^{\prime}(N)$, completing the proof.

We immediately see that $\mathscr{A}$ amenable implies that $\mathscr{A}^{\prime \prime}$ is Connes-amenable. Furthermore, if $\mathscr{A}$ is itself a dual Banach algebra, then Corollary 3.5 shows that if $\mathscr{A}^{\prime \prime}$ is Connes-amenable, then $\mathscr{A}$ is Connes-amenable: notice that if $e_{\mathscr{A}}$ is the unit of $\mathscr{A}^{\prime \prime}$, then

$$
\begin{aligned}
\left\langle\kappa_{\mathscr{A}_{*}}^{\prime}\left(e_{\mathscr{A}}^{\prime \prime}\right) a, \mu\right\rangle & =\left\langle e_{\mathscr{A}^{\prime \prime}} \cdot a, \kappa_{\mathscr{A}_{*}}(\mu)\right\rangle \\
& =\left\langle\kappa_{\mathscr{A}}(a), \kappa_{\mathscr{A}_{*}}(\mu)\right\rangle=\langle a, \mu\rangle \quad\left(a \in \mathscr{A}, \mu \in \mathscr{A}_{*}\right),
\end{aligned}
$$

so that $\kappa_{\mathscr{A}_{*}}^{\prime}\left(e_{\mathscr{A}}^{\prime \prime}\right)$ is the unit of $\mathscr{A}$.

\section{Injectivity of the predual module}

Let $\mathscr{A}$ be a Banach algebra, and let $E$ and $F$ be Banach left $\mathscr{A}$-modules. We write $\mathscr{A} \mathscr{B}(E, F)$ for the closed subspace of $\mathscr{B}(E, F)$ consisting of left $\mathscr{A}$ module homomorphisms, and similarly write $\mathscr{B}_{\mathscr{A}}(E, F)$ and $\mathscr{A}_{\mathscr{B}}(E, F)$ for right $\mathscr{A}$-module and $\mathscr{A}$-bimodule homomorphisms, respectively. We say that $T \in \mathscr{A} \mathscr{B}(E, F)$ is admissible if both the kernel and image of $T$ are closed, complemented subspaces of, respectively, $E$ and $F$. If $T$ is injective, this is equivalent to the existence of $S \in \mathscr{B}(F, E)$ such that $S T=I_{E}$.

Definition 4.1. Let $\mathscr{A}$ be a Banach algebra, and let $E$ be a Banach left $\mathscr{A}$ module. Then $E$ is injective if, whenever $F$ and $G$ are Banach left $\mathscr{A}$-modules, $\theta \in \mathscr{A} \mathscr{B}(F, G)$ is injective and admissible, and $\sigma \in \mathscr{A} \mathscr{B}(F, E)$, there exists $\rho \in \mathscr{A} \mathscr{B}(G, E)$ with $\rho \circ \theta=\sigma$.

We say that $E$ is left-injective when we wish to stress that we are treating $E$ as a left module. Similar definitions hold for right modules and bimodules (written right-injective and bi-injective where necessary).

Let $\mathscr{A}$ be a Banach algebra, let $E$ be a Banach left $\mathscr{A}$-module, and turn $\mathscr{B}(\mathscr{A}, E)$ into a left $\mathscr{A}$-module by setting

$$
(a \cdot T)(b)=T(b a) \quad(a, b \in \mathscr{A}, T \in \mathscr{B}(\mathscr{A}, E)) .
$$

Then there is a canonical left $\mathscr{A}$-module homomorphism $\iota: E \rightarrow \mathscr{B}(\mathscr{A}, E)$ given by

$$
\iota(x)(a)=a \cdot x \quad(a \in \mathscr{A}, x \in E) .
$$

Notice that if $E$ is a closed submodule of $\mathscr{A}^{\prime}$, then $\mathscr{B}(\mathscr{A}, E)$ is a closed submodule of $(A \widehat{\otimes} A)^{\prime}=\mathscr{B}\left(\mathscr{A}, \mathscr{A}^{\prime}\right)$, and $\iota$ is the restriction of $\Delta_{\mathscr{A}}^{\prime}: \mathscr{A}^{\prime} \rightarrow$ $\mathscr{B}\left(\mathscr{A}, \mathscr{A}^{\prime}\right)$ to $E$. 
Similarly, we turn $\mathscr{B}(\mathscr{A} \widehat{\otimes} \mathscr{A}, E)$ into a Banach $\mathscr{A}$-bimodule by

$$
\begin{aligned}
(a \cdot T)(b \otimes c)=T(b a \otimes c), \quad(T \cdot a)(b \otimes c) & =T(b \otimes a c) \\
(a, b, c & \in \mathscr{A}, T \in \mathscr{B}(\mathscr{A} \widehat{\otimes} \mathscr{A}, E)) .
\end{aligned}
$$

We then define (with an abuse of notation) $\iota: E \rightarrow \mathscr{B}(\mathscr{A} \widehat{\otimes} \mathscr{A}, E)$ by

$$
\iota(x)(a \otimes b)=a \cdot x \cdot b \quad(x \in E, a \otimes b \in \mathscr{A} \widehat{\otimes} \mathscr{A}),
$$

so that $\iota$ is an $\mathscr{A}$-bimodule homomorphism.

We can also turn $\mathscr{B}(\mathscr{A}, E)$ into a right $\mathscr{A}$-module by reversing the above (in particular, we need to take the other possible choice in Section 3 leading to different module actions as compared to those in (1).)

Proposition 4.2. Let $\mathscr{A}$ be a Banach algebra, and let E be a faithful Banach left $\mathscr{A}$-module (that is, for each non-zero $x \in E$ there exists $a \in \mathscr{A}$ with $a \cdot x \neq 0)$. Then $E$ is injective if and only if there exists $\phi \in \mathscr{A} \mathscr{B}(\mathscr{B}(\mathscr{A}, E), E)$ such that $\phi \circ \iota=I_{E}$.

Similarly, if $E$ is a left and right faithful Banach $\mathscr{A}$-bimodule (that is, for each non-zero $x \in E$ there exists $a, b \in \mathscr{A}$ with $a \cdot x \neq 0$ and $x \cdot b \neq 0$ ). Then $E$ is injective if and only if there exists $\phi \in \mathscr{A} \mathscr{B} \mathscr{A}(\mathscr{B}(\mathscr{A} \widehat{\otimes} \mathscr{A}, E), E)$ such that $\phi \circ \iota=I_{E}$.

Proof. The first claim is [4, Proposition 1.7], and the second claim is an obvious generalisation.

Again, there exists a similar characterisation for right modules. The above result is useful, as it allows us to work with $\mathscr{A}$ and not its unitisation (which is the usual approach).

Let $\mathscr{A}$ be a dual Banach algebra with predual $\mathscr{A}_{*}$. It is simple to show (see [13]) that if $\mathscr{A}_{*}$ is bi-injective, then $\mathscr{A}$ is Connes-amenable. Helemskii showed in [8] that for a von Neumann algebra $\mathscr{A}$, the converse is true. However, Runde (see [13]) and Tabaldyev (see [15]) have shown that $M(G)$, the measure algebra of a locally compact group $G$, while being a dual Banach algebra with predual $C_{0}(G)$, has that $C_{0}(G)$ is a left-injective $M(G)$-module only when $G$ is finite. Recall that Runde (see [11]) has shown that $M(G)$ is Connes-amenable if and only if $G$ is amenable.

Similarly, it is simple to show (using a virtual diagonal) that if $\mathscr{A}$ is a Banach algebra with a bounded approximate identity, then $\mathscr{A}$ is amenable if and only if $\mathscr{A}^{\prime}$ is bi-injective.

Let $E$ and $F$ be Banach left $\mathscr{A}$-modules, and let $\phi: E \rightarrow F$ be a left $\mathscr{A}$-module homomorphism which is bounded below. Then $\phi(E)$ is a closed 
submodule of $F$, so that $F / \phi(E)$ is a Banach left $\mathscr{A}$-module. Hence we have a short exact sequence:

$$
0 \longrightarrow E \stackrel{\phi}{\longleftrightarrow-\bar{P}^{-}} F \longrightarrow F / \phi(E) \longrightarrow 0 \text {. }
$$

If there exists a bounded linear map $P: F \rightarrow E$ such that $P \circ \phi=I_{E}$, then we say that the short exact sequence is admissible. If, further, we may choose $P$ to be a left $\mathscr{A}$-module homomorphism, then the short exact sequence is said to split. Similar definitions hold for right modules and bimodules.

Proposition 4.3. Let $\mathscr{A}$ be a Banach algebra, let $E$ be a Banach left $\mathscr{A}$ module, and consider the following short exact sequence:

$$
0 \longrightarrow E \stackrel{\iota}{\longleftrightarrow---} \mathscr{P}(\mathscr{A}, E) \longrightarrow \mathscr{B}(\mathscr{A}, E) / \iota(E) \longrightarrow 0
$$

Then $E$ is injective if and only if this short exact sequence is admissible and splits.

Proof. See, for example, [14, Section 5.3]. Notice that when $\mathscr{A}$ is unital, the short exact sequence is certainly admissible.

Proposition 4.4. Let $\mathscr{A}$ be a unital dual Banach algebra with predual $\mathscr{A}_{*}$, and consider the following admissible short exact sequence of $\mathscr{A}$-bimodules:

$$
\begin{aligned}
0 \longrightarrow \mathscr{A}_{*} \frac{\Delta_{\mathscr{A}}^{\prime}}{\longrightarrow-\bar{P}^{--}} & \sigma W C\left((\mathscr{A} \widehat{\otimes} \mathscr{A})^{\prime}\right) \\
& \longrightarrow \sigma W C\left((\mathscr{A} \widehat{\otimes} \mathscr{A})^{\prime}\right) / \Delta_{\mathscr{A}}^{\prime}\left(\mathscr{A}_{*}\right) \longrightarrow 0 .
\end{aligned}
$$

Then $\mathscr{A}$ is Connes-amenable if and only if this short exact sequence splits.

Proof. Notice that $\Delta_{\mathscr{A}}^{\prime}$ certainly maps $\mathscr{A}_{*}$ into $\sigma W C\left((\mathscr{A} \widehat{\otimes} \mathscr{A})^{\prime}\right)=$ $\sigma W C\left(\mathscr{B}\left(\mathscr{A}, \mathscr{A}^{\prime}\right)\right)$, and that Corollary 3.5 shows that we can define $P$ : $\sigma W C\left(\mathscr{B}\left(\mathscr{A}, \mathscr{A}^{\prime}\right)\right) \rightarrow \mathscr{A}_{*}$ by $P(T)=T\left(e_{\mathscr{A}}\right)$ for $T \in \sigma W C\left(\mathscr{B}\left(\mathscr{A}, \mathscr{A}^{\prime}\right)\right)$.

Suppose that we can choose $P$ to be an $\mathscr{A}$-bimodule homomorphism. Then let $M=P^{\prime}\left(e_{\mathscr{A}}\right)$, so that for $a \in \mathscr{A}$ and $T \in \sigma W C\left(\mathscr{B}\left(\mathscr{A}, \mathscr{A}^{\prime}\right)\right)$,

$$
\langle a \cdot M-M \cdot a, T\rangle=\left\langle e_{\mathscr{A}}, P(T \cdot a-a \cdot T)\right\rangle=\langle a-a, P(T)\rangle=0,
$$

so that $a \cdot M-M \cdot a$. Also $\Delta_{\mathscr{A}}^{\prime \prime}(M)=\left(P \circ \Delta_{\mathscr{A}}^{\prime}\right)^{\prime}\left(e_{\mathscr{A}}\right)=e_{\mathscr{A}}$, so that $M$ is a $\sigma W C$-virtual diagonal, and hence $\mathscr{A}$ is Connes-amenable by Runde's theorem.

Conversely, let $M$ be a $\sigma W C$-virtual diagonal and define $P: \sigma W C(\mathscr{B}(\mathscr{A}$, $\left.\left.\mathscr{A}^{\prime}\right)\right) \rightarrow \mathscr{A}^{\prime}$ by

$$
\langle P(T), a\rangle=\langle M, a \cdot T\rangle \quad\left(a \in \mathscr{A}, T \in \sigma W C\left(\mathscr{B}\left(\mathscr{A}, \mathscr{A}^{\prime}\right)\right) .\right.
$$


Let $\left(a_{\alpha}\right)$ be a bounded net in $\mathscr{A}$ which tends to $a \in \mathscr{A}$ in the $\sigma\left(\mathscr{A}, \mathscr{A}_{*}\right)$ topology. By definition, $a_{\alpha} \cdot T \rightarrow a \cdot T$ weakly, for each $T \in \sigma W C\left(\mathscr{B}\left(\mathscr{A}, \mathscr{A}^{\prime}\right)\right)$, so that $\left\langle P(T), a_{\alpha}\right\rangle \rightarrow\langle P(T), a\rangle$. This implies that $P$ maps into $\mathscr{A}_{*}$, as required. Then, for $\mu \in \mathscr{A}_{*}$,

$$
\begin{aligned}
\left\langle a, P \Delta_{\mathscr{A}}^{\prime}(\mu)\right\rangle=\left\langle M, a \cdot \Delta_{\mathscr{A}}^{\prime}(\mu)\right\rangle & =\left\langle M, \Delta_{\mathscr{A}}^{\prime}(a \cdot \mu)\right\rangle \\
& =\left\langle e_{\mathscr{A}}, a \cdot \mu\right\rangle=\langle a, \mu\rangle \quad(a \in \mathscr{A}),
\end{aligned}
$$

so that $P \Delta_{\mathscr{A}}^{\prime}=I_{\mathscr{A}_{*}}$. Finally, we note that

$$
\begin{aligned}
\langle P(a \cdot T \cdot b), c\rangle & =\langle M, c a \cdot T \cdot b\rangle=\langle b \cdot M, c a \cdot T\rangle \\
& =\langle M \cdot b, c a \cdot T\rangle=\langle P(T), b c a\rangle \\
& =\langle a \cdot P(T) \cdot b, c\rangle \quad\left(a, b, c \in \mathscr{A}, T \in \sigma W C\left(\mathscr{B}\left(\mathscr{A}, \mathscr{A}^{\prime}\right)\right)\right),
\end{aligned}
$$

so that $P$ is an $\mathscr{A}$-bimodule homomorphism, as required.

Let $\mathscr{A}$ be an Arens regular Banach algebra. By reversing the argument Theorem 3.8, we can show that $\Delta_{\mathscr{A}}^{\prime}: \mathscr{A}^{\prime} \rightarrow \mathscr{B}\left(\mathscr{A}, \mathscr{A}^{\prime}\right)$ actually maps into WAP $\left(\mathscr{W}\left(\mathscr{A}, \mathscr{A}^{\prime}\right)\right)$. Furthermore, if $\mathscr{A}^{\prime \prime}$ is unital, then we may define $P$ : $\mathrm{WAP}\left(\mathscr{W}\left(\mathscr{A}, \mathscr{A}^{\prime}\right)\right) \rightarrow \mathscr{A}^{\prime}$ by

$$
\langle P(T), a\rangle=\left\langle e_{\mathscr{A}^{\prime \prime}}, P(a)\right\rangle \quad\left(a \in \mathscr{A}, T \in \operatorname{WAP}\left(\mathscr{W}\left(\mathscr{A}, \mathscr{A}^{\prime}\right)\right)\right) .
$$

Then we have that

$$
\left\langle P \Delta_{\mathscr{A}}^{\prime}(\mu), a\right\rangle=\left\langle e_{\mathscr{A}}^{\prime \prime}, a \cdot \mu\right\rangle=\langle\mu, a\rangle \quad\left(a \in \mathscr{A}, \mu \in \mathscr{A}^{\prime}\right) .
$$

Proposition 4.5. Let $\mathscr{A}$ be an Arens regular Banach algebra such that $\mathscr{A}^{\prime \prime}$ is unital, and consider the following admissible short exact sequence of $\mathscr{A}$-bimodules:

(3) $0 \longrightarrow \mathscr{A}^{\prime} \frac{\Delta_{\mathscr{A}}^{\prime}}{\leftarrow-\frac{-}{P}--} \operatorname{WAP}\left(\mathscr{W}\left(\mathscr{A}, \mathscr{A}^{\prime}\right)\right)$

$$
\operatorname{WAP}\left(\mathscr{W}\left(\mathscr{A}, \mathscr{A}^{\prime}\right)\right) / \Delta_{\mathscr{A}}^{\prime}\left(\mathscr{A}^{\prime}\right) \longrightarrow 0 .
$$

Then $\mathscr{A}^{\prime \prime}$ is Connes-amenable if and only if this short exact sequence splits.

Proof. This follows in the same manner as the above proof, using Theorem 3.9 . 


\section{Beurling algebras}

Let $S$ be a discrete semigroup (we can extend the following definitions to locally compact semigroups, but for the questions we are interested in, the results for non-discrete groups are trivial). A weight on $S$ is a function $\omega: S \rightarrow \mathrm{R}_{+}$such that

$$
\omega(s t) \leq \omega(s) \omega(t) \quad(s, t \in S) .
$$

Furthermore, if $S$ is unital with unit $u_{S}$, then we also insist that $\omega\left(u_{S}\right)=1$. This last condition is simply a normalisation condition, as we can always set $\hat{\omega}(s)=\sup \left\{\omega(s t) \omega(t)^{-1}: t \in S\right\}$ for each $s \in S$. For $s, t \in S$, we have that $\omega(s t) \leq \hat{\omega}(s) \omega(t)$, so that

$$
\begin{aligned}
\hat{\omega}(s t)=\sup \left\{\omega(s t r) \omega(r)^{-1}\right. & : r \in S\} \\
\leq & \sup \left\{\hat{\omega}(s) \omega(t r) \omega(r)^{-1}: r \in S\right\}=\hat{\omega}(s) \hat{\omega}(t) .
\end{aligned}
$$

Clearly $\hat{\omega}\left(u_{S}\right)=1$ and $\hat{\omega}(s) \leq \omega(s)$ for each $s \in S$, while $\hat{\omega}(s) \geq$ $\omega(s) \omega\left(u_{S}\right)^{-1}$, so that $\hat{\omega}$ is equivalent to $\omega$.

We form the Banach space

$$
l^{1}(S, \omega)=\left\{\left(a_{g}\right)_{g \in S} \subseteq \mathrm{C}:\left\|\left(a_{g}\right)\right\|:=\sum_{g \in S}\left|a_{g}\right| \omega(g)<\infty\right\} .
$$

Then $l^{1}(S, \omega)$, with the convolution product, is a Banach algebra, called a Beurling algebra. See [1] and [3] for further information on Beurling algebras and, in particular, their second duals.

It will be more convenient for us to think of $l^{1}(S, \omega)$ as the Banach space $l^{1}(S)$ together with a weighted algebra product. Indeed, for $g \in S$, let $\delta_{g} \in$ $l^{1}(S)$ be the standard unit vector basis element which is thought of as a pointmass at $g$. Then each $x \in l^{1}(S)$ can be written uniquely as $x=\sum_{g \in S} x_{g} \delta_{g}$ for some family $\left(x_{g}\right) \subseteq \mathrm{C}$ such that $\|x\|=\sum_{g \in S}\left|x_{g}\right|<\infty$. We then define

$$
\Omega(g, h)=\frac{\omega(g h)}{\omega(g) \omega(h)}, \quad \delta_{g} \star_{\omega} \delta_{h}=\delta_{g} \star \delta_{h}=\delta_{g h} \Omega(g, h) \quad(g, h \in S),
$$

and extend $\star$ to $l^{1}(S)$ by linearity and continuity.

For example, if $\omega$ and $\hat{\omega}$ are equivalent weights on $S$, then define $\psi$ : $l^{1}(S, \omega) \rightarrow l^{1}(S, \hat{\omega})$ by $\psi\left(\delta_{s}\right)=\hat{\omega}(s) \omega(s)^{-1} \delta_{s}$. As $\omega$ and $\hat{\omega}$ are equivalent, $\psi$ is an isomorphism of Banach spaces. Then $\psi\left(\delta_{s} \star \delta_{t}\right)=$ $\omega(s t) \omega(s)^{-1} \omega(t)^{-1} \hat{\omega}(s t) \omega(s t)^{-1} \delta_{s t}=\psi\left(\delta_{s}\right) \star \psi\left(\delta_{t}\right)$, so that $\psi$ is a homomorphism. 
For a set $I$, we define the spaces $c_{0}(I)$ and $l^{\infty}(I)$ in the standard way. We write $\left(e_{i}\right)_{i \in I}$ for the standard unit vector basis of $c_{0}(I)$ (or its image in $l^{\infty}(I)$ ), so that $\left\langle\delta_{j}, e_{i}\right\rangle=\delta_{i, j}$, the Kronecker delta, for $j \in I$.

For a semigroup $S$ and $s \in S$, we define maps $L_{s}, R_{s}: S \rightarrow S$ by

$$
L_{s}(t)=s t, \quad R_{s}(t)=t s \quad(t \in S) .
$$

If, for each $s \in S, L_{s}$ and $R_{s}$ are finite-to-one maps, then we say that $S$ is weakly cancellative. When $L_{s}$ and $R_{s}$ are injective for each $s \in S$, we say that $S$ is cancellative. When $S$ is abelian and cancellative, a construction going back to Grothendieck shows that $S$ is a sub-semigroup of some abelian group. However, this can fail to hold for non-abelian semigroups.

Proposition 5.1. Let $S$ be a weakly cancellative semigroup, let $\omega$ be a weight on $S$, and let $\mathscr{A}=l^{1}(S, \omega)$. Then $c_{0}(S) \subseteq l^{\infty}(S)=\mathscr{A}^{\prime}$ is a sub- $\mathscr{A}$ module of $\mathscr{A}^{\prime}$, so that $l^{1}(S, \omega)$ is a dual Banach algebra with predual $c_{0}(S)$.

Proof. For $g, h \in S$ and $a=\left(a_{s}\right)_{s \in S} \in l^{1}(S, \omega)$, we have

$$
\left\langle e_{g} \cdot \delta_{h}, a\right\rangle=\left\langle e_{g}, \delta_{h} \star a\right\rangle=\left\langle e_{g}, \sum_{s \in S} a_{s} \delta_{h s} \Omega(h, s)\right\rangle=\sum_{\{s \in S: h s=g\}} a_{s} \Omega(h, s) .
$$

As $S$ is weakly cancellative, there exists at most finitely many $s \in S$ such that $h s=g$, so that $e_{g} \cdot \delta_{h}$ is a member of $c_{0}(S)$. Thus we see that $c_{0}(S)$ is a right sub- $\mathscr{A}$-module of $\mathscr{A}^{\prime}$. The argument on the left follows in an analogous manner.

Notice that the above result will hold for some semigroups $S$ which are not weakly cancellative, provided that the weight behaves in a certain way. However, it would appear that the later results in this section do not easily generalise to the non-weakly cancellative case.

Following [3, Definition 2.2], we have the following definition.

Definition 5.2. Let $I$ and $J$ be non-empty infinite sets, and let $f: I \times J \rightarrow$ $C$ be a function. Then $f$ clusters on $I \times J$ if

$$
\lim _{n \rightarrow \infty} \lim _{m \rightarrow \infty} f\left(x_{m}, y_{n}\right)=\lim _{m \rightarrow \infty} \lim _{n \rightarrow \infty} f\left(x_{m}, y_{n}\right),
$$

whenever $\left(x_{m}\right) \subseteq I$ and $\left(y_{n}\right) \subseteq J$ are sequences of distinct elements, and both iterated limits exist.

Furthermore, $f 0$-clusters on $I \times J$ if $f$ clusters on $I \times J$, and the iterated limits are always 0 , when they exist.

From now on we shall exclude the trivial case when our (semi-)group is finite. 
THEOREM 5.3. Let $S$ be a discrete, weakly cancellative semigroup, and let $\omega$ be a weight on $S$. Then the following are equivalent:

(1) $l^{1}(S, \omega)$ is Arens regular;

(2) for sequences of distinct elements $\left(g_{j}\right)$ and $\left(h_{k}\right)$ in $S$, we have

$$
\lim _{j \rightarrow \infty} \lim _{k \rightarrow \infty} \Omega\left(g_{j}, h_{k}\right)=0,
$$

whenever the iterated limit exists;

(1) $\Omega$ 0-clusters on $S \times S$.

PRoOF. That (1) and (2) are equivalent for cancellative semigroups is [1, Theorem 1]. Close examination of the proof shows that this holds for weakly cancellative semigroups as well. That (1) and (3) are equivalent follows by generalising the proof of [3, Theorem 7.11], which is essentially an application of Grothendieck's criterion for an operator to be weakly compact. Alternatively, it follows easily that (2) and (3) are equivalent by considering the opposite semigroup to $S$ where we reverse the product.

In [1] it is also shown that if $G$ is a discrete, uncountable group, then $l^{1}(G, \omega)$ is not Arens regular for any weight $\omega$. Furthermore, by [1, Theorem 2], if $G$ is a non-discrete locally compact group, then $L^{1}(G, \omega)$ is never Arens regular.

We shall consider both the Connes-amenability of $l^{1}(S, \omega)^{\prime \prime}$ and $l^{1}(S, \omega)$ (with respect to the canonical predual $c_{0}(S)$ ) as, with reference to Corollary 3.5 and Theorem 3.8, the calculations should be similar.

Proposition 5.4. Let I be a non-empty set, and let $X \subseteq l^{\infty}(I)$ be a subset. Then the following are equivalent:

(1) $X$ is relatively weakly compact;

(2) $X$ is relatively sequentially weakly compact;

(3) the absolutely convex hull of $X$ is relatively weakly compact;

(4) if we define $f: I \times X \rightarrow \mathrm{C}$ by $f(i, x)=\left\langle x, \delta_{i}\right\rangle$ for $i \in I$ and $x \in X$, then $f$ clusters on $I \times X$;

Proof. That (1) and (2) are equivalent is the Eberlien-Smulian theorem; that (1) and (3) are equivalent is the Krein-Smulian theorem. That (1) and (4) are equivalent is a result of Grothendieck, detailed in, for example, [3, Theorem 2.3].

It is standard that for non-empty sets $I$ and $J$, we have that $l^{1}(I) \widehat{\otimes} l^{1}(J)=$ $l^{1}(I \times J)$, where, for $i \in I$ and $j \in J, \delta_{i} \otimes \delta_{j} \in l^{1}(I) \widehat{\otimes} l^{1}(J)$ is identified with $\delta_{(i, j)} \in l^{1}(I \times J)$. Thus we have $\left(l^{1}(I) \widehat{\otimes} l^{1}(J)\right)^{\prime}=\mathscr{B}\left(l^{1}(I), l^{\infty}(J)\right)=$ 
$l^{1}(I \times J)^{\prime}=l^{\infty}(I \times J)$, where $T \in \mathscr{B}\left(l^{1}(I), l^{\infty}(J)\right)$ is identified with $\left(T_{(i, j)}\right) \in l^{\infty}(I \times J)$, where $T_{(i, j)}=\left\langle T\left(\delta_{i}\right), \delta_{j}\right\rangle$.

For a non-empty set $I$, the unit ball of $l^{1}(I)$ is the closure of the absolutelyconvex hull of the set $\left\{\delta_{i}: i \in I\right\}$, so that for a Banach space $E$, by the Krein-Smulian theorem, a map $T: l^{1}(I) \rightarrow E$ is weakly compact if and only if the set $\left\{T\left(\delta_{i}\right): i \in I\right\}$ is relatively weakly compact in $E$.

Proposition 5.5. Let $S$ be a weakly cancellative semigroup, let $\omega$ be a weight on $S$, and let $\mathscr{A}=l^{1}(S, \omega)$. Let $T \in \mathscr{B}\left(\mathscr{A}, \mathscr{A}^{\prime}\right)$ be such that $T(\mathscr{A}) \subseteq$ $\kappa_{c_{0}(S)}\left(c_{0}(S)\right)$ and $T^{\prime}\left(\kappa_{\mathscr{A}}(\mathscr{A})\right) \subseteq \kappa_{c_{0}(S)}\left(c_{0}(S)\right)$. Then $T \in \mathscr{W}\left(\mathscr{A}, \mathscr{A}^{\prime}\right)$, and $T \in$ $\mathrm{WAP}\left(\mathscr{W}\left(\mathscr{A}, \mathscr{A}^{\prime}\right)\right)$ if and only if, for each sequence $\left(k_{n}\right)$ of distinct elements of $S$, and each sequence $\left(g_{m}, h_{m}\right)$ of distinct elements of $S \times S$ such that the repeated limits

$$
\begin{array}{ll}
\lim _{n} \lim _{m}\left\langle T\left(\delta_{h_{m}}\right), \delta_{k_{n} g_{m}}\right\rangle, & \lim _{n} \lim _{m} \Omega\left(k_{n}, g_{m}\right) \\
\lim _{n} \lim _{m}\left\langle T\left(\delta_{h_{m} k_{n}}\right), \delta_{g_{m}}\right\rangle, & \lim _{n} \lim _{m} \Omega\left(h_{m}, k_{n}\right)
\end{array}
$$

all exist, we have that at least one repeated limit in each row is zero.

Proof. That $T$ is weakly compact follows from Gantmacher's Theorem (compare with Corollary 3.5). To show that $T \in$ WAP, by Lemma 3.4, we are required to show that the maps $\phi_{r}$ and $\phi_{l}$ are weakly compact. We shall show that $\phi_{l}$ is weakly compact if and only if one of the repeated limits in the first line (4) is zero; the proof that $\phi_{r}$ is related to (5) follows in a similar way. We have that

$$
\phi_{l}\left(\delta_{(g, h)}\right)=\phi_{l}\left(\delta_{g} \otimes \delta_{h}\right)=\delta_{g} \cdot T\left(\delta_{h}\right) \quad(g, h \in S) .
$$

By Proposition 5.4, $\phi_{l}$ is weakly compact if and only if the function

$$
\begin{aligned}
S \times(S \times S) \rightarrow \mathrm{C} ; \quad(k,(g, h)) \mapsto & \left\langle\delta_{g} \cdot T\left(\delta_{h}\right), \delta_{k}\right\rangle \\
& =\left\langle T\left(\delta_{h}\right), \delta_{k g}\right\rangle \Omega(k, g) \quad(g, h, k \in S)
\end{aligned}
$$

clusters on $S \times(S \times S)$. As $T$ is weakly compact, the function

$$
S \times S \rightarrow \mathrm{C} ; \quad(g, h) \mapsto\left\langle T\left(\delta_{g}\right), \delta_{h}\right\rangle \quad(g, h \in S)
$$

does cluster on $S \times S$.

Let $\left(k_{n}\right)$ be a sequence of distinct elements of $S$, and let $\left(g_{m}, h_{m}\right)$ be a sequence of distinct elements of $S \times S$ such that the iterated limits

(6) $\quad \lim _{n} \lim _{m}\left\langle T\left(\delta_{h_{m}}\right), \delta_{k_{n} g_{m}}\right\rangle \Omega\left(k_{n}, g_{m}\right), \quad \lim _{m} \lim _{n}\left\langle T\left(\delta_{h_{m}}\right), \delta_{k_{n} g_{m}}\right\rangle \Omega\left(k_{n}, g_{m}\right)$ 
exist. We now investigate when these iterated limits are equal.

Suppose that $\left(g_{m}\right)$ has a constant subsequence, so we may suppose that $g_{m}=g$ for all $m$. By moving to a subsequence if necessary, we may also suppose that $\lim _{n} \Omega\left(k_{n}, g\right)=\alpha$, say, and that $\left(k_{n} g\right)$ is a sequence of distinct elements (as $S$ is weakly cancellative). Then

$$
\begin{aligned}
\lim _{n} \lim _{m}\left\langle T\left(\delta_{h_{m}}\right), \delta_{k_{n} g_{m}}\right\rangle \Omega\left(k_{n}, g_{m}\right) & =\lim _{n} \Omega\left(k_{n}, g\right) \lim _{m}\left\langle T\left(\delta_{h_{m}}\right), \delta_{k_{n} g}\right\rangle \\
& =\alpha \lim _{n} \lim _{m}\left\langle T\left(\delta_{h_{m}}\right), \delta_{k_{n} g}\right\rangle \\
& =\alpha \lim _{m} \lim _{n}\left\langle T\left(\delta_{h_{m}}\right), \delta_{k_{n} g}\right\rangle \\
& =\lim _{m} \lim _{n}\left\langle T\left(\delta_{h_{m}}\right), \delta_{k_{n} g_{m}}\right\rangle \Omega\left(k_{n}, g_{m}\right),
\end{aligned}
$$

where we can swap the order of taking limits, as $T$ is weakly compact.

Alternatively, if we cannot move to a subsequence such that $\left(g_{m}\right)$ is constant, then we may move to subsequence such that $\left(g_{m}\right)$ is a sequence of distinct elements, and such that the iterated limits

$$
\begin{array}{cl}
\lim _{m} \lim _{n} \Omega\left(k_{n}, g_{m}\right), & \lim _{n} \lim _{m} \Omega\left(k_{n}, g_{m}\right), \\
\lim _{m} \lim _{n}\left\langle T\left(\delta_{h_{m}}\right), \delta_{k_{n} g_{m}}\right\rangle, & \lim _{n} \lim _{m}\left\langle T\left(\delta_{h_{m}}\right), \delta_{k_{n} g_{m}}\right\rangle
\end{array}
$$

all exists. As $T(\mathscr{A}) \subseteq \kappa_{c_{0}(S)}\left(c_{0}(S)\right)$, we have that

$$
\left\{g \in S:\left|\left\langle T\left(\delta_{h}\right), \delta_{g}\right\rangle\right| \geq \epsilon\right\} \text { is finite } \quad(\epsilon>0, h \in S) .
$$

Consequently, and using the fact that $S$ is weakly cancellative, we see that

$$
\lim _{n}\left\langle T\left(\delta_{h_{m}}\right), \delta_{k_{n} g_{m}}\right\rangle=0
$$

for each $m$. Hence the iterated limits in (6) are equal if and only if we have that at least one repeated limit in (4) is zero.

Proposition 5.6. Let $S$ be a discrete, unital, weakly cancellative semigroup, and let $\omega$ be a weight on $S$ such that $\mathscr{A}=l^{1}(S, \omega)$ is Arens regular. Then $\operatorname{WAP}\left(\mathscr{W}\left(\mathscr{A}, \mathscr{A}^{\prime}\right)\right)=\mathscr{W}\left(\mathscr{A}, \mathscr{A}^{\prime}\right)$.

Proof. Let $T \in \mathscr{W}\left(\mathscr{A}, \mathscr{A}^{\prime}\right)$. We can follow the above proof through until the point at which we use the fact that $T(\mathscr{A}) \subseteq \kappa_{c_{0}(S)}\left(c_{0}(S)\right)$. However, as $l^{1}(S, \omega)$ is Arens regular, by Theorem 5.3, we have that

$$
\lim _{m} \lim _{n} \Omega\left(k_{n}, g_{m}\right)=\lim _{n} \lim _{m} \Omega\left(k_{n}, g_{m}\right)=0,
$$

so that the iterated limits in (6) must be 0 , implying that $\phi_{l}$ is weakly compact. In a similar manner, $\phi_{r}$ is weakly compact. 
THEOREM 5.7. Let $S$ be a discrete weakly cancellative semigroup, and let $\omega$ be a weight on $S$ such that $\mathscr{A}=l^{1}(S, \omega)$ is Arens regular and $\mathscr{A}^{\prime \prime}$ is unital with unit e. $\mathscr{A}^{\prime \prime}$. Then $\mathscr{A}^{\prime \prime}$ is Connes-amenable if and only if there exists $M \in(\mathscr{A} \widehat{\otimes} \mathscr{A})^{\prime \prime}=l^{\infty}(S \times S)^{\prime}$ such that:

(1) $\left\langle M,\left(f_{g h} \Omega(g, h)\right)_{(g, h) \in S \times S}\right\rangle=\left\langle e_{\mathscr{A}}\right.$,,$\left.f\right\rangle$ for each bounded family $\left(f_{g}\right)_{g \in S}$;

(2) $\left\langle M,(f(h k, g) \Omega(h, k)-f(h, k g) \Omega(k, g))_{(g, h) \in S \times S}\right\rangle=0$ for each $k \in S$, and each bounded function $f: S \times S \rightarrow C$ which clusters on $S \times S$.

Proof. We use Theorem 3.9 and Proposition 5.6. For $f=\left(f_{g}\right)_{g \in S} \in$ $l^{\infty}(S)$, we have

$$
\left\langle\Delta_{\mathscr{A}}^{\prime}(f), \delta_{g} \otimes \delta_{h}\right\rangle=\left\langle f, \delta_{g h}\right\rangle \Omega(g, h) \quad(g, h \in S),
$$

so that $\Delta_{\mathscr{A}}^{\prime}(f)=\left(\left\langle f, \delta_{g h}\right\rangle \Omega(g, h)\right)_{(g, h) \in S \times S} \in l^{\infty}(S \times S)$. As $f \in l^{\infty}(S)$ was arbitrary, we have condition (1).

For $T \in \mathscr{B}\left(\mathscr{A}, \mathscr{A}^{\prime}\right)$, we treat $T$ as being a member of $l^{\infty}(S \times S)$. Then $T$ is weakly compact if and only if the family $\left(\left\langle T\left(\delta_{g}\right), \delta_{h}\right\rangle\right)_{(g, h) \in S \times S}$ clusters on $S \times S$. For $k \in S$, we have

$$
\left\langle\delta_{k} \cdot T-T \cdot \delta_{k}, \delta_{g} \otimes \delta_{h}\right\rangle=\left\langle T\left(\delta_{h k}\right), \delta_{g}\right\rangle \Omega(h, k)-\left\langle T\left(\delta_{h}\right), \delta_{k g}\right\rangle \Omega(k, g) .
$$

Thus we have condition (2).

Notice that if $S$ is unital with unit $u_{S}$, then the unit of $\mathscr{A}$ (and hence $\mathscr{A}^{\prime \prime}$ ) is $\delta_{u_{S}}$. In this case, condition (1) reduces to $\left\langle M,\left(f_{g h} \Omega(g, h)\right)_{(g, h) \in S \times S}\right\rangle=f_{u_{S}}$.

THEOREM 5.8. Let $S$ be a discrete unital semigroup, let $\omega$ be a weight on $S$, and let $\mathscr{A}=l^{1}(S, \omega)$. Then $\mathscr{A}$ is amenable if and only if there exists $M \in(\mathscr{A} \widehat{\otimes} \mathscr{A})^{\prime \prime}=l^{\infty}(S \times S)^{\prime}$ such that:

(1) $\left\langle M,\left(f_{g h} \Omega(g, h)\right)_{(g, h) \in S \times S}\right\rangle=f_{u_{S}}$, where $u_{S} \in S$ is the unit of $S$, for each bounded family $\left(f_{g}\right)_{g \in S}$;

(2) $\left\langle M,(f(h k, g) \Omega(h, k)-f(h, k g) \Omega(k, g))_{(g, h) \in S \times S}\right\rangle=0$ for each $k \in S$, and each bounded function $f: S \times S \rightarrow \mathrm{C}$.

Proof. This follows as above, but by using a virtual diagonal in place of a $\sigma W C$-virutal diagonal.

Notice that condition (2) of Theorem 5.8 is strictly stronger than condition (2) of Theorem 5.7.

THEOREM 5.9. Let $S$ be a discrete, weakly cancellative semigroup, let $\omega$ be a weight on $S$, and let $\mathscr{A}=l^{1}(S, \omega)$ be unital with unit $e_{\mathscr{A}}$. Then $\mathscr{A}$ is Connes-amenable, with respect to the predual $c_{0}(S)$, if and only if there exists $M \in(\mathscr{A} \widehat{\otimes} \mathscr{A})^{\prime \prime}=l^{\infty}(S \times S)^{\prime}$ such that: 
(1) $\left\langle M,\left(f_{g h} \Omega(g, h)\right)_{(g, h) \in S \times S}\right\rangle=\left\langle e_{\mathscr{A}}, f\right\rangle$ for each family $\left(f_{g}\right)_{g \in S} \in c_{0}(S)$;

(2) $\left\langle M,(f(h k, g) \Omega(h, k)-f(h, k g) \Omega(k, g))_{(g, h) \in S \times S}\right\rangle=0$ for each $k \in S$, and each bounded function $f: S \times S \rightarrow \mathrm{C}$ which is such that the map $T \in \mathscr{B}\left(\mathscr{A}, \mathscr{A}^{\prime}\right)$, defined by $\left\langle T\left(\delta_{g}\right), \delta_{h}\right\rangle=f(g, h)$ for $g, h \in S$, satisfies the conclusions of Proposition 5.5.

Proof. We now use Theorem 3.6.

We shall now characterise when $l^{1}(S, \omega)$ and $l^{1}(S, \omega)^{\prime \prime}$ are Connes-amenable in terms of properties of $S$ and $\omega$. For a discrete group $G$, a weight $\omega$ on $G$ and $h \in G$, define $J_{h} \in \mathscr{B}\left(l^{\infty}(G)\right)$ by

$$
\begin{aligned}
J_{h}(f)=\left(f_{h g} \Omega(h, g) \omega(h) \Omega\left(g^{-1}, h^{-1}\right) \omega\left(h^{-1}\right)\right)_{g \in G} & \\
& \left(f=\left(f_{g}\right)_{g \in G} \in l^{\infty}(G)\right) .
\end{aligned}
$$

Notice then that, for $f \in l^{\infty}(G)$, we have

$$
\left\|J_{h}(f)\right\|=\sup _{g}\left|f_{h g}\right| \omega(h g) \omega(g)^{-1} \omega\left(g^{-1} h^{-1}\right) \omega\left(g^{-1}\right)^{-1} \leq\|f\| \omega(h) \omega\left(h^{-1}\right),
$$

so that $J_{h}$ is bounded. Define $\hat{\omega}(g)=\omega(g) \omega\left(g^{-1}\right)$ for $g \in G$, so that $\hat{\omega}$ is the auxiliary weight considered in [6]. If we identify $l^{\infty}(G)$ with $l^{\infty}\left(G, \hat{\omega}^{-1}\right)$ by the map $\left(x_{g}\right)_{g \in G} \mapsto\left(x_{g} \hat{\omega}(g)^{-1}\right)_{g \in G}$, then $J_{h}$ is simply the operator induced by left-translation by $h$ on $l^{\infty}\left(G, \hat{\omega}^{-1}\right)$.

Definition 5.10. Let $G$ be a discrete group, and let $\omega$ be a weight on $G$. We say that $G$ is $\omega$-amenable if there exists $N \in l^{\infty}(G)^{\prime}$ such that:

(1) $\left\langle N,\left(\Omega\left(g, g^{-1}\right)\right)_{g \in G}\right\rangle=1$, where $\Omega$ is defined by $\omega$, and hence $\left(\Omega\left(g, g^{-1}\right)\right)_{g \in G}$ is a bounded family forming an element of $l^{\infty}(G)$;

(2) $J_{h}^{\prime}(N)=N$ for each $h \in G$.

By the remark above about $J_{h}$, we see that $G$ is $\omega$-amenable if and only if $l^{\infty}(G, \omega)$ has a non-zero, left-invariant mean. Notice that if $\omega$ is identically 1 , then this condition reduces to the usual notion of a group being amenable (we usually require that $N$ is a mean, in that $N$ is a positive functional on $l^{\infty}(G)$, but by forming real and imaginary parts, and then positive and negative parts, we can easily generate a non-zero scalar multiple of a mean from a functional $N$ satisfying the definition above).

THEOREM 5.11. Let $G$ be a discrete group, let $\omega$ be a weight on $G$, and let $\mathscr{A}=l^{1}(G, \omega)$. Then the following are equivalent:

(1) $\mathscr{A}$ is Connes-amenable, with respect to the predual $c_{0}(G)$;

(2) $\mathscr{A}$ is amenable; 
(3) $G$ is $\omega$-amenable.

Furthermore, if $\mathscr{A}$ is Arens regular, then these conditions are equivalent to $\mathscr{A}^{\prime \prime}$ being Connes-amenable.

Proof. It is clear that (2) implies (1). When $\mathscr{A}$ is Arens regular, (2) implies that $\mathscr{A}^{\prime \prime}$ is Connes-amenable, and $\mathscr{A}^{\prime \prime}$ Connes-amenable implies (1). We shall thus show that (1) implies (3). That (3) implies (2) follows from the equivalence of (3) and (2) established in [6, Theorem 3.2].

If (1) holds, then let $M \in l^{\infty}(G \times G)^{\prime}$ be given as in Theorem 5.9. Define $\phi: l^{\infty}(G) \rightarrow l^{\infty}(G \times G)$ by

$$
\left\langle\phi(f), \delta_{(g, h)}\right\rangle=\left\{\begin{array}{ll}
f_{g} & : g=h^{-1}, \\
0 & : g \neq h^{-1},
\end{array} \quad\left(f=\left(f_{g}\right)_{g \in G} \in l^{\infty}(G)\right),\right.
$$

and let $N=\phi^{\prime}(M) \in l^{\infty}(G)^{\prime}$. Then we have

$\phi\left(\left(\Omega\left(g, g^{-1}\right)\right)_{g \in G}\right)=\left(\delta_{h, g^{-1}} \Omega(g, h)\right)_{(g, h) \in G \times G}=\left(\delta_{g h, e_{G}} \Omega(g, h)\right)_{(g, h) \in G \times G}$,

where $\delta$ is the Kronecker delta, so that

$$
\left\langle N,\left(\Omega\left(g, g^{-1}\right)\right)_{g \in G}\right\rangle=\delta_{e_{G}, e_{G}}=1,
$$

by condition (1) on $M$ from Theorem 5.9; clearly $\left(\delta_{e_{G}, g}\right)_{g \in G} \in c_{0}(G)$.

Fix $k \in G$ and $f \in l^{\infty}(G)$. Define $F: G \times G \rightarrow C$ by

$$
F(h, g)=\delta_{g h, k} f_{g} \omega(k) \omega\left(h k^{-1}\right) \omega(h)^{-1} . \quad(g, h \in G) .
$$

Then we have $|F(h, g)| \leq\left|f_{g}\|\omega(k)\| \omega\left(h k^{-1}\right)\right||\omega(h)|^{-1} \leq$ $\|f\|_{\infty}|\omega(k)|\left|\omega\left(k^{-1}\right)\right|$, so that $F$ is bounded. Let $T: \mathscr{A} \rightarrow \mathscr{A}^{\prime}$ be the operator associated with $F$. For $g, h \in G$, we have that $F(h, g) \neq 0$ only when $g h=k$, so that $T(\mathscr{A}) \subseteq c_{0}(S)$ and $T^{\prime}\left(\kappa_{\mathscr{A}}(\mathscr{A})\right) \subseteq c_{0}(S)$. Furthermore, if $\left(k_{n}\right)$ is a sequence of distinct elements in $G$, and $\left(g_{m}, h_{m}\right)$ is a sequence of distinct elements in $G \times G$, then $\lim _{n} \lim _{m} F\left(h_{m}, k_{n} g_{m}\right)=0$. This follows, as for $n_{0}$ fixed, $k_{n_{0}} g_{m} h_{m}=k$ only if $g_{m} h_{m}=k_{n_{0}}^{-1} k$, so if this holds for all sufficiently large $m$, we have that $k_{n} g_{m} h_{m} \neq k$ for sufficiently large $m$ and $n \neq n_{0}$. Similarly, $\lim _{n} \lim _{m} F\left(h_{m} k_{n}, g_{m}\right)=0$, so that $F$ satisfies the conditions of Proposition 5.5.

Notice that

$$
\begin{aligned}
\left\langle\phi\left(J_{k}(f)\right), \delta_{(g, h)}\right\rangle & =\delta_{g h, e_{G}}\left\langle J_{k}(f), \delta_{g}\right\rangle \\
& =\delta_{g h, e_{G}} f_{k g} \omega(k g) \omega(g)^{-1} \omega\left(g^{-1} k^{-1}\right) \omega\left(g^{-1}\right)^{-1} .
\end{aligned}
$$


Thus we have

$$
\begin{aligned}
& F(h k, g) \Omega(h, k)-F(h, k g) \Omega(k, g) \\
&= \delta_{g h k, k} f_{g} \omega(k) \omega\left(h k k^{-1}\right) \omega(h k)^{-1} \Omega(h, k) \\
& \quad-\delta_{k g h, k} f_{k g} \omega(k) \omega\left(h k^{-1}\right) \omega(h)^{-1} \Omega(k, g) \\
&= \delta_{g h, e_{G}} f_{g}-\delta_{g h, e_{G}} f_{k g} \omega\left(h k^{-1}\right) \omega(h)^{-1} \omega(k g) \omega(g)^{-1} \\
&=\left\langle\phi(f)-\phi\left(J_{k}(f)\right), \delta_{(g, h)}\right\rangle .
\end{aligned}
$$

So, by condition (2) from Theorem 5.9, we have that

$$
\left\langle N, f-J_{k}(f)\right\rangle=0,
$$

which, as $f$ was arbitrary, shows that $N=J_{k}^{\prime}(N)$, as required.

EXAMPLE 5.12. If $S$ is a semigroup which is not cancellative, then it is possible for $l^{1}(S)$ to be unital while $S$ is not. For example, let $S$ be $\mathrm{Z}$ with adjoined idempotents $u$ and $v$ such that $u v=v u=0$ and $u n=n u=v n=$ $n v=n$ for $n \in \mathrm{Z}$. Then $S$ is a weakly cancellative, commutative semigroup without a unit, but $e=\delta_{u}+\delta_{v}-\delta_{0}$ is easily seen to be a unit for $l^{1}(S)$. Indeed, $S$ is seen to be a finite semilattice of groups, so by the result of [5], $l^{1}(S)$ is amenable.

In [6, Theorem 2.3] it is shown that if $l^{1}(S, \omega)$ is amenable for a cancellative, unital semigroup $S$ and some weight $\omega$, then $S$ is actually a group. We shall now show that this holds for Connes-amenability as well.

THEOREM 5.13. Let $S$ be a weakly cancellative semigroup, let $\omega$ be a weight on $S$, and let $\mathscr{A}=l^{1}(S, \omega)$. Suppose that $\mathscr{A}$ is Connes-amenable with respect to the predual $c_{0}(S)$. If $S$ is cancellative or unital, then $S$ is a group.

Proof. As $\mathscr{A}$ is Connes-amenable, let $M \in(\mathscr{A} \widehat{\otimes} \mathscr{A})^{\prime \prime}$ be as in Theorem 5.9. Then $\mathscr{A}$ is unital, with unit $e_{\mathscr{A}}=\left(a_{s}\right)_{s \in S} \in l^{1}(S, \omega)$ say. For now, we shall not assume that $e_{\mathscr{A}}$ has norm one, as the standard renorming to ensure this will not (a priori) necessarily yield an $l^{1}(S, \hat{\omega})$ algebra for some weight $\hat{\omega}$. Suppose now that $S$ is cancellative. We see that

$\sum_{s \in S} a_{s} \delta_{s h} \Omega(s, h)=e_{\mathscr{A}} \star \delta_{h}=\delta_{h}=\delta_{h} \star e_{\mathscr{A}}=\sum_{s \in S} a_{s} \delta_{h s} \Omega(h, s) \quad(h \in S)$.

In particular, for each $h \in S$ there is a unique (as $S$ is cancellative) $u_{h} \in S$ such that $h u_{h}=h$ (so that $h u_{h} h=h^{2}$ implying that $u_{h} h=h$ ), and we have that $a_{u_{h}} \omega\left(u_{h}\right)^{-1}=1$. We also see that $a_{s}=0$ for each $s \in S$ such that $s h \neq h$, 
that is, $s \neq u_{h}$. However, $h$ was arbitrary, so that $S$ is unital with unit $u_{S}$, and $e_{\mathscr{A}}=\omega\left(u_{S}\right) \delta_{u_{S}}$, where we can now assume that $\omega\left(u_{S}\right)=1$ by a renorming.

Now suppose that $S$ is a unital, weakly cancellative semigroup, so that the unit of $\mathscr{A}$ is $\delta_{u_{S}}$. Suppose that $s \in S$ has no right inverse. Define $F: S \times S \rightarrow C$ by

$$
F(h, s g)=0, \quad F(h s, g)=\left\{\begin{array}{ll}
\Omega(g, h s) & : g h=u_{S}, \\
0 & : \text { otherwise. }
\end{array} \quad(g, h \in S) .\right.
$$

To show that this is well-defined, suppose that for $g, h, j, k \in S$, we have that $h=j s, s g=k$ and $k j=u_{S}$. Then $s(g j)=k j=u_{S}$, so that $s$ has a right inverse, a contradiction. Then $F$ is bounded, so let $T: \mathscr{A} \rightarrow \mathscr{A}^{\prime}$ be the operator associated with $F$. Then $F(a, b) \neq 0$ only when $b a=s$, so as $S$ is weakly cancellative, we see that $T(\mathscr{A}) \subseteq c_{0}(S)$ and $T^{\prime}\left(\kappa_{\mathscr{A}}(\mathscr{A})\right) \subseteq c_{0}(S)$.

Suppose that for sequences of distinct elements $\left(k_{n}\right) \subseteq S$ and $\left(g_{m}, h_{m}\right) \subseteq$ $S \times S$, we have that

$$
\lim _{n} \lim _{m}\left\langle T\left(\delta_{h_{m}}\right), \delta_{k_{n} g_{m}}\right\rangle=\lim _{n} \lim _{m} F\left(h_{m}, k_{n} g_{m}\right) \neq 0 .
$$

Then, for some $N>0$ and $\epsilon>0$, for each $n \geq N$, $\lim _{m} F\left(h_{m}, k_{n} g_{m}\right) \geq$ $\epsilon$. Hence, for $n \geq N$, there exists $M_{n}>0$ such that if $m \geq M_{n}$, then $k_{n} g_{m} h_{m}=s$ (as otherwise $F\left(h_{m}, k_{n} g_{m}\right)=0$ ). This, however, contradicts $S$ being weakly cancellative. Similarly, if $\lim _{n} \lim _{m}\left\langle T\left(\delta_{h_{m} k_{n}}\right), \delta_{g_{m}}\right\rangle \neq 0$, then we need $g_{m} h_{m} k_{n}=s$ for all $n, m$ sufficiently large, which is a contradiction. Thus $T$ satisfies all the conditions of Proposition 5.5.

Then, for $g, h \in S$, if $g h=u_{S}$, we have that $\Omega(h, s) \Omega(g, h s)=$ $\omega(h)^{-1} \omega(g)^{-1}=\Omega(g, h)$, so that

$$
F(h s, g) \Omega(h, s)-F(h, s g) \Omega(s, g)= \begin{cases}\Omega(g, h) & : g h=u_{S}, \\ 0 & : \text { otherwise. }\end{cases}
$$

Hence condition (2) of Theorem 5.9 implies that $\left\langle M,\left(\delta_{g h, u_{S}} \Omega(g, h)\right)_{(g, h) \in S \times S}\right\rangle$ $=0$, which contradicts condition (1) of this theorem. Hence every element of $S$ has a right inverse.

By symmetry (or by repeating the argument on the left) we see that every element of $S$ has a left inverse, and that hence $S$ must be a group.

We hence have the following theorem, which shows that weighted semigroup algebras behave like $\mathrm{C}^{*}$-algebras with regards to Connes-amenability.

THEOREM 5.14. Let $S$ be a discrete cancellative semigroup, and let $\omega$ be a weight on $S$. The following are equivalent:

(1) $l^{1}(S, \omega)$ is amenable; 
(2) $l^{1}(S, \omega)$ is Connes-amenable, with respect to the predual $c_{0}(S)$; If $l^{1}(S, \omega)$ is Arens regular, then these conditions are equivalent to $l^{1}(S, \omega)^{\prime \prime}$ being Connes-amenable. These equivalent conditions imply that $S$ is a group.

This result extends the result of [12], where it is shown that $M(G)$, the measure algebra of a locally compact group $G$, is Connes-amenable if and only if $G$ is amenable. This follows as, for discrete groups $G, M(G)=l^{1}(G)$.

Example 5.15. Let $\omega$ be the weight on $Z$ defined by $\omega(n)=1+|n|$ for $n \in \mathrm{Z}$. By Theorem 5.3, $\mathscr{A}=l^{1}(\mathrm{Z}, \omega)$ is Arens regular. For $m, n \in \mathbf{Z}$ and $f=\left(a_{k}\right)_{k \in Z} \in l^{\infty}(\mathbf{Z})$, we have that

$$
\left\langle\delta_{m} \cdot f, \delta_{n}\right\rangle=\left\langle f, \delta_{n+m} \Omega(n, m)\right\rangle=f_{n+m} \frac{1+|n+m|}{(1+|n|)(1+|m|)} .
$$

Suppose that $M \square \kappa_{\mathscr{A}}\left(\delta_{m}\right)=\kappa_{\mathscr{A}}(a)$ for some $m \in \mathbf{Z}, M \in l^{\infty}(\mathrm{Z})^{\prime}$ and $a \in \mathscr{A}$. Then $\left\langle M, \delta_{m} \cdot f\right\rangle=\langle f, a\rangle$ for each $f \in l^{\infty}(\mathbf{Z})$, so by letting $f=\kappa_{c_{0}(\mathrm{Z})}\left(e_{k}\right) \in$ $c_{0}(\mathbf{Z})$, we see that $a=\sum_{k \in Z} a_{k} \delta_{k}$, where $a_{k}=\left\langle M, \delta_{m} \cdot \kappa_{c_{0}(\mathrm{Z})}\left(e_{k}\right)\right\rangle$. However, $\delta_{m} \cdot \kappa_{c_{0}(\mathbf{Z})}\left(e_{k}\right) \in \kappa_{c_{0}(\mathbf{Z})}\left(c_{0}(\mathbf{Z})\right)$ for each $k \in \mathbf{Z}$, so if $M \in c_{0}(\mathbf{Z})^{\circ}$, then $a=0$.

Consequently, if $M \square \kappa_{\mathscr{A}}\left(\delta_{m}\right) \in \kappa_{\mathscr{A}}(\mathscr{A})$ for each $m \in \mathbf{Z}$ and $M \in l^{\infty}(\mathrm{Z})^{\prime}$, then $\delta_{m} \cdot f \in \kappa_{c_{0}(\mathbf{Z})}\left(c_{0}(\mathbf{Z})\right)$ for each $m \in \mathbf{Z}$ and $f \in l^{\infty}(\mathbf{Z})$. However, if $\mathbf{1} \in l^{\infty}(Z)$ is the constant 1 sequence, then

$$
\lim _{n}\left\langle\delta_{m} \cdot \mathbf{1}, \delta_{n}\right\rangle=\lim _{n} \frac{1+|n+m|}{(1+|n|)(1+|m|)}=\frac{1}{1+|m|},
$$

so that $\delta_{m} \cdot \mathbf{1} \notin \kappa_{c_{0}}(\mathrm{Z})\left(c_{0}(\mathrm{Z})\right)$.

We hence conclude that $\mathscr{A}$ is not an ideal in $\mathscr{A}^{\prime \prime}$, and so we cannot apply Theorem 1.3 in this case.

Unfortunately, for the viewpoint of finding a counter-example to the conjecture that $\mathscr{A}$ is amenable if and only if $\mathscr{A}^{\prime \prime}$ is Connes-amenable, it is not possible for $l^{1}(S, \omega)$ to be both amenable and Arens regular.

THEOREM 5.16. Let $G$ be discrete group, and let $\omega$ be a weight on $G$. Then $l^{1}(G, \omega)$ is amenable if and only if $G$ is an amenable group, and $\sup \left\{\omega(g) \omega\left(g^{-1}\right): g \in G\right\}<\infty$.

Proof. This is [6, Theorem 3.2].

Proposition 5.17. Let $S$ be a discrete, unital semigroup, and let $\omega$ be a weight on $S$ such that $\mathscr{A}=l^{1}(S, \omega)$ is Arens regular. Let $K>0$ and $B \subseteq S$ be such that for each $g \in B, g$ has a right inverse $g^{-1}$ (which need not be unique), and $\omega(g) \omega\left(g^{-1}\right) \leq K$. Then $B$ is finite. 
Proof. For $g \in B$ and $h \in S$, we have

$$
\omega(g) \omega(h)=\omega(g) \omega\left(h g g^{-1}\right) \leq \omega(g) \omega(h g) \omega\left(g^{-1}\right) \leq K \omega(h g),
$$

so that $\Omega(h, g) \geq K^{-1}$. Suppose now that $B$ is infinite. Then we can easily construct sequences which violate condition (2) of Theorem 5.3, showing that $\mathscr{A}$ is not Arens regular. This contradiction shows that $B$ must be finite.

\subsection{Injectivity of the predual module}

Let $S$ be a unital, weakly cancellative semigroup, let $\omega$ be a weight on $S$, and let $\mathscr{A}=l^{1}(S, \omega), \mathscr{A}_{*}=c_{0}(S)$. Then $\mathscr{B}\left(\mathscr{A}, \mathscr{A}_{*}\right)=\mathscr{B}\left(l^{1}, c_{0}\right)=l^{\infty}\left(c_{0}\right) \subseteq$ $l^{\infty}(S \times S)$, where we identify $T: \mathscr{A} \rightarrow \mathscr{A}_{*}$ with the bounded family $\left(\left\langle\delta_{s}, T\left(\delta_{t}\right)\right\rangle\right)_{(s, t) \in S \times S}$. Let $\phi: \mathscr{B}\left(\mathscr{A}, \mathscr{A}_{*}\right) \rightarrow \mathscr{A}_{*}$, so that $\phi$ is represented by a bounded family $\left(M_{s}\right)_{s \in S} \subseteq \mathscr{B}\left(\mathscr{A}, \mathscr{A}_{*}\right)^{\prime}$ using the relation

$$
\left\langle\delta_{s}, \phi(T)\right\rangle=\left\langle M_{s}, T\right\rangle \quad\left(s \in S, T \in \mathscr{B}\left(\mathscr{A}, \mathscr{A}_{*}\right)\right) .
$$

Suppose further that $\phi$ is a left $\mathscr{A}$-module homomorphism. Then

$$
\begin{aligned}
\left\langle\delta_{s}, \phi(T)\right\rangle=\left\langle\delta_{u_{s}}, \phi\left(\delta_{s} \cdot T\right)\right\rangle & =\left\langle M_{u_{s}}, \delta_{s} \cdot T\right\rangle \\
& =\left\langle M_{s}, T\right\rangle \quad\left(s \in S, T \in \mathscr{B}\left(\mathscr{A}, \mathscr{A}_{*}\right)\right),
\end{aligned}
$$

so that $M_{s}=M_{u_{S}} \cdot \delta_{s}$ for each $s \in S$. We see also that $\phi$ maps into $c_{0}(S)$ (and not just $\left.l^{\infty}(S)\right)$ if and only if

$$
\lim _{s \rightarrow \infty}\left\langle M_{u_{S}}, \delta_{s} \cdot T\right\rangle=0 \quad\left(T \in \mathscr{B}\left(\mathscr{A}, \mathscr{A}_{*}\right)\right) .
$$

Conversely, if condition (7) holds, then for $s, t \in S$ and $T \in \mathscr{B}\left(\mathscr{A}, \mathscr{A}_{*}\right)$, we have that

$$
\begin{aligned}
\left\langle\delta_{s}, \phi\left(\delta_{t} \cdot T\right)\right\rangle & =\left\langle M_{s}, \delta_{t} \cdot T\right\rangle=\left\langle M_{u_{S}}, \delta_{s} \cdot \delta_{t} \cdot T\right\rangle=\Omega(s, t)\left\langle M_{s t}, T\right\rangle \\
& =\Omega(s, t)\left\langle\delta_{s t}, \phi(T)\right\rangle=\left\langle\delta_{s}, \delta_{t} \cdot \phi(T)\right\rangle .
\end{aligned}
$$

Hence $\phi$ is a left $\mathscr{A}$-module homomorphism.

Notice that $c_{0}(S \times S) \subseteq \mathscr{B}\left(\mathscr{A}, \mathscr{A}_{*}\right)$, so that $c_{0}(S \times S)^{\circ} \subseteq \mathscr{B}\left(\mathscr{A}, \mathscr{A}_{*}\right)^{\prime}$.

Definition 5.18. Let $G$ be a group and $\omega$ be a weight on $G$ such that for each $\epsilon>0$, the set $\left\{g \in G: \omega(g) \omega\left(g^{-1}\right)<\epsilon^{-1}\right\}$ is finite. Then we say that the weight $\omega$ is strongly non-amenable.

Proposition 5.19. Let $G$ be a group, let $\omega$ be a weight on $G$ such that $\omega$ is not strongly non-amenable, and let $\phi: \mathscr{B}\left(\mathscr{A}, c_{0}(G)\right) \rightarrow c_{0}(G)$ be a left $\mathscr{A}$-module homomorphism. If $\phi$ is represented by $\left(M_{g}\right)_{g \in G}$ as above, then $M_{u_{G}} \in c_{0}(S \times S)^{\circ}$. 
Proof. We adapt the methods of [4] to the weighted, discrete case. As $\omega$ is not strongly non-amenable, there exists some $K>0$ such that the set $X_{K}=\left\{g \in G: \omega(g) \omega\left(g^{-1}\right) \leq K\right\}$ is infinite. Let $M=M_{u_{G}}$, and suppose that $M \notin c_{0}(G \times G)^{\circ}$, so that for some $g, h \in G$, we have that $\delta:=\left\langle M, e_{(g, h)}\right\rangle \neq 0$. We shall henceforth treat $e_{(g, h)}$ as a member of $\mathscr{B}\left(\mathscr{A}, c_{0}(G)\right)$, noting that for $k \in G$,

$$
\left\langle\delta_{s},\left(\delta_{k} \cdot e_{(g, h)}\right)\left(\delta_{t}\right)\right\rangle= \begin{cases}\Omega(t, k) & : s=g, t=h k^{-1}, \\ 0 & : \text { otherwise. }\end{cases}
$$

We claim that we can find a sequence $\left(g_{n}\right)_{n \in \mathbf{N}}$ of distinct elements in $G$ such that

$$
\begin{aligned}
\left|\left\langle M \cdot \delta_{g_{m}^{-1} g_{n}}, e_{(g, h)}\right\rangle\right| & \leq K^{-1} 2^{-2-|m-n|} \quad(n \neq m), \\
\omega\left(g_{n}\right) \omega\left(g_{n}^{-1}\right) & \leq K \quad(n \in \mathrm{N}) .
\end{aligned}
$$

We can do this as $\phi$ must map into $c_{0}(G)$, so that for any $T: \mathscr{A} \rightarrow c_{0}(G)$, we have $\lim _{g \rightarrow \infty}\left\langle M \cdot \delta_{g}, T\right\rangle=0$. Explicitly, let $g_{1} \in X_{K}$ be arbitrary, and suppose that we have found $g_{1}, \ldots, g_{k}$. Then notice that the sets

$$
\begin{aligned}
& \left\{s \in G:\left|\left\langle M \cdot \delta_{s^{-1} g_{n}}, e_{(g, h)}\right\rangle\right|>K^{-1} 2^{-2-|k+1-n|}: 1 \leq n \leq k\right\}, \\
& \left\{s \in G:\left|\left\langle M \cdot \delta_{g_{m}^{-1} s}, e_{(g, h)}\right\rangle\right|>K^{-1} 2^{-2-|k+1-m|}: 1 \leq m \leq k\right\}
\end{aligned}
$$

are finite, so as $X_{K}$ is infinite, we can certainly find some $x_{k+1}$.

Then, for $x=\left(x_{n}\right) \in l^{\infty}(\mathrm{N})$, define $T_{x}: \mathscr{A} \rightarrow c_{0}(G)$ by setting $\left\langle\delta_{g}, T_{x}\left(\delta_{h g_{n}^{-1}}\right)\right\rangle=x_{n} \Omega\left(h g_{n}^{-1}, g_{n}\right)$ for $n \geq 1$, and $\left\langle\delta_{s}, T_{x}\left(\delta_{t}\right)\right\rangle=0$ otherwise. Then clearly $T_{x}$ does map into $c_{0}(G)$, and $\left\|T_{x}\right\| \leq\|x\|$. Notice that for $s, t \in G$, we have

$$
\begin{aligned}
\left\langle\delta_{s}, T_{x}\left(\delta_{t}\right)\right\rangle & = \begin{cases}x_{n} \Omega\left(t, g_{n}\right) & : s=g, t=h g_{n}^{-1}, \\
0 & : \text { otherwise, }\end{cases} \\
& =\sum_{n} x_{n}\left\langle\delta_{s},\left(\delta_{g_{n}} \cdot e_{(g, h)}\right)\left(\delta_{t}\right)\right\rangle .
\end{aligned}
$$

Define $Q: l^{\infty}(\mathrm{N}) \rightarrow c_{0}(\mathrm{~N})$ by

$$
\left\langle\delta_{n}, Q(x)\right\rangle=\left\langle M, \delta_{g_{n}^{-1}} \cdot T_{x}\right\rangle \quad(n \in \mathrm{N}),
$$

so that $Q$ is bounded and linear.

Let $n_{0} \geq 1$ and let $x=e_{n_{0}} \in c_{0}(\mathrm{~N}) \subseteq l^{\infty}(\mathrm{N})$. Then, $T_{x}=\delta_{g_{n_{0}}} \cdot e_{(g, h)}$, so that

$$
\begin{aligned}
\left\langle\delta_{n}, Q(x)\right\rangle & =\left\langle M, \delta_{g_{n}^{-1}} \cdot T_{x}\right\rangle=\left\langle M, \delta_{g_{n}^{-1}} \cdot\left(\delta_{g_{n_{0}}} \cdot e_{(g, h)}\right)\right\rangle \\
& = \begin{cases}\delta \Omega\left(g_{n_{0}}^{-1}, g_{n_{0}}\right) & : n=n_{0}, \\
\Omega\left(g_{n}^{-1}, g_{n_{0}}\right)\left\langle M \cdot e_{g_{n}^{-1} g_{n_{0}}}, e_{(g, h)}\right\rangle & : n \neq n_{0} .\end{cases}
\end{aligned}
$$


Define $Q_{1} \in \mathscr{B}\left(c_{0}(\mathrm{~N})\right)$ by

$$
Q_{1}(x)=\left(\Omega\left(g_{n}^{-1}, g_{n}\right) x_{n}\right)_{n \in \mathrm{N}} \quad\left(x=\left(x_{n}\right) \in c_{0}(\mathrm{~N})\right) .
$$

Then, as each $g_{n} \in X_{K}, Q_{1}$ is an invertible operator. Let $Q_{2}$ be the restriction of $Q$ to $c_{0}(\mathrm{~N})$, so that $Q_{2} \in \mathscr{B}\left(c_{0}(\mathrm{~N})\right)$ and $Q_{2}=\delta Q_{1}+\delta Q_{3} Q_{1}$ for some $Q_{3} \in \mathscr{B}\left(c_{0}(\mathrm{~N})\right)$. Thus $Q_{3}=\delta^{-1} Q_{2} Q_{1}^{-1}-I_{c_{0}(\mathrm{~N})}$, so that for $x \in c_{0}(\mathrm{~N})$, we have that

$$
\begin{aligned}
\left\|Q_{3}(x)\right\| & =\sup _{n}\left|\left\langle\delta_{n}, \delta^{-1} Q_{2} Q_{1}^{-1}(x)-x\right\rangle\right| \\
& =\sup _{n}\left|\sum_{m} x_{m}\left\langle\delta_{n}, \delta^{-1} Q_{2} Q_{1}^{-1}\left(e_{m}\right)-e_{m}\right\rangle\right| \\
& =\sup _{n}\left|\sum_{m \neq n} x_{m} \Omega\left(g_{m}^{-1}, g_{m}\right)^{-1} \Omega\left(g_{n}^{-1}, g_{m}\right)\left\langle M \cdot \delta_{g_{n}^{-1} g_{m}}, e_{(g, h)}\right\rangle\right| \\
& \leq K^{-1} \sup _{n} \sum_{m \neq n}\left|x_{m}\right| 2^{-2-|m-n|} \omega\left(g_{m}\right) \omega\left(g_{m}^{-1}\right) \leq\|x\| / 2 .
\end{aligned}
$$

Consequently $Q_{3}-I_{c_{0}(\mathrm{~N})}$ is invertible, so that $Q_{2} Q_{1}^{-1}$ is invertible, showing that $Q_{2}$ is invertible. However, this implies that $Q_{2}^{-1} Q: l^{\infty}(\mathrm{N}) \rightarrow c_{0}(\mathrm{~N})$ is a projection, which is a well-known contradiction, completing the proof.

THEOREM 5.20. Let $G$ be a countable group, let $\omega$ be a weight which is not strongly non-amenable, and let $\mathscr{A}=l^{1}(G, \omega)$. Then $c_{0}(G)$ is not left-injective.

Proof. Suppose, towards a contradiction, that $c_{0}(G)$ is left-injective, so that there exists $M=M_{u_{G}} \in \mathscr{B}\left(\mathscr{A}, \mathscr{A}_{*}\right)^{\prime}$ as above, with the additional condition that

$$
\begin{aligned}
\delta_{g, h} & =\left\langle\delta_{g}, \phi \Delta_{\mathscr{A}}^{\prime}\left(e_{h}\right)\right\rangle=\left\langle M, \delta_{g} \cdot \Delta_{\mathscr{A}}^{\prime}\left(e_{h}\right)\right\rangle=\Omega\left(h g^{-1}, g\right)\left\langle M, \Delta_{\mathscr{A}}^{\prime}\left(e_{h g^{-1}}\right)\right\rangle \\
& =\Omega\left(h g^{-1}, g\right)\left\langle M,\left(\delta_{s t, h g^{-1}} \Omega(s, t)\right)_{(s, t) \in G \times G}\right\rangle \quad(g, h \in G) .
\end{aligned}
$$

This clearly reduces to

$$
\delta_{g, u_{G}}=\left\langle M,\left(\delta_{s t, g} \Omega(s, t)\right)_{(s, t) \in G \times G}\right\rangle \quad(g \in G) .
$$

As $G$ is countable, we can enumerate $G$ as $G=\left\{g_{n}: n \in \mathrm{N}\right\}$. Then, for $g_{n} \in G$, let $X_{g_{n}}=\left\{g_{1}, \ldots, g_{n}\right\} \subseteq G$. Define $Q: l^{\infty}(G) \rightarrow \mathscr{B}\left(\mathscr{A}, c_{0}(G)\right)$ by

$$
\left\langle\delta_{s}, Q(x)\left(\delta_{t}\right)\right\rangle=\Omega(s, t) \sum_{g \in X_{t}} x_{g} \delta_{s t, g} \quad\left(s, t \in G, x \in l^{\infty}(G)\right) .
$$


Then, for each $t \in G$, as $X_{t}$ is finite, we see that $Q(x)\left(\delta_{t}\right) \in c_{0}(G)$, so $Q$ is well-defined. Clearly $Q$ is linear, and we see that for $x \in l^{\infty}(G)$,

$$
\|Q(x)\|=\sup _{s, t \in G} \Omega(s, t)\left|\sum_{g \in X_{t}} x_{g} \delta_{s t, g}\right| \leq \sup _{s, t \in G} \sum_{\left\{g \in X_{t}: g=s t\right\}}\left|x_{g}\right|=\|x\|,
$$

so that $Q$ is norm-decreasing. Then, for $h \in G$, we have that

$$
\left\langle\delta_{s}, Q\left(e_{h}\right)\left(\delta_{t}\right)\right\rangle=\Omega(s, t) \sum_{g \in X_{t}} \delta_{g, h} \delta_{s t, g}= \begin{cases}\left\langle\delta_{s}, \Delta_{\mathscr{A}}^{\prime}\left(e_{h}\right)\left(\delta_{t}\right)\right\rangle & : h \in X_{t}, \\ 0 & : h \notin X_{t} .\end{cases}
$$

Let $h=g_{n_{0}}$, so that $\left\{t \in G: h \notin X_{t}\right\}=\left\{g_{n} \in G: h \notin X_{g_{n}}\right\}=$ $\left\{g_{1}, g_{2}, \ldots, g_{n_{0}-1}\right\}$. We hence see that $Q\left(e_{g_{0}}\right)-\Delta_{\mathscr{A}}^{\prime}\left(e_{g_{0}}\right) \in c_{0}(G \times G)$. By the preceding proposition, we hence have that $I_{c_{0}(G)}=\phi \circ \Delta_{\mathscr{A}}^{\prime}=\phi \circ\left(\left.Q\right|_{c_{0}(G)}\right)$. However, this implies that $\phi \circ Q: l^{\infty}(G) \rightarrow c_{0}(G)$ is a projection onto $c_{0}(G)$, giving us the required contradiction.

We have not been able to establish if $c_{0}(S)$ can ever be a left-injective $l^{1}(S, \omega)$-module for some semigroup $S$ and weight $\omega$. However, Helemskii's theorem about Connes-amenable von Neumann algebras does not hold for weighted semigroup algebras.

THeORem 5.21. Let $S$ be a discrete, weakly cancellative semigroup, let $\omega$ be a weight on $S$, and let $\mathscr{A}=l^{1}(S, \omega)$. When $S$ is unital, or $S$ is cancellative, $c_{0}(S)$ is not a bi-injective $\mathscr{A}$-bimodule.

Proof. Suppose, towards a contradiction, that $c_{0}(S)$ is bi-injective. Then $\mathscr{A}$ is Connes-amenable, so that Theorem 5.14 implies that $\mathscr{A}$ is amenable, and that $S=G$ is an amenable group. By [16, Corollary 2] there exists a positive character $\phi$ on $G$ (that is, a group homomorphism to the multiplicative group of positive reals) such that $\phi$ is equivalent to $\omega$. Thus $l^{1}(G, \omega)$ is isomorphic to $l^{1}(G, \phi)$, and as $\phi$ is multiplicative, it follows that $l^{1}(G, \phi)$ is simply $l^{1}(G)$ (as the $\Omega$ associated with $\phi$ is identically 1$)$. The result now follows from results in [4].

\section{Open questions}

We state a few open questions of interest:

(1) Let $\mathscr{A}$ be an Arens regular Banach algebra such that $\mathscr{A}^{\prime \prime}$ is Connesamenable. Need $\mathscr{A}$ be amenable?

(2) This is true for $\mathrm{C}^{*}$-algebras. Can we find a "simple" proof? 
(3) Let $\mathscr{A}$ be a dual Banach algebra with predual $\mathscr{A}_{*}$, and suppose that $\mathscr{A}_{*}$ is bi-injective. Is $\mathscr{A}$ necessarily a von Neumann algebra or the bidual of an Arens regular Banach algebra $\mathscr{B}$ such that $\mathscr{B}$ is an ideal in $\mathscr{A}$ ?

(4) Let $S$ be a (weakly cancellative) semigroup, and let $\omega$ be a weight on $S$. Classify (up to isomorphism) the preduals of $l^{1}(S, \omega)$, and calculate which preduals yield a Connes-amenable Banach algebra.

(5) This question was asked by Niels Grønbæk. In most of our examples, it is obvious that when $\mathscr{A}$ is a Connes-amenable dual Banach algebra, there is $\mathscr{B} \subseteq \mathscr{A}$ which is weak*-dense and amenable. Is this always true?

ACKNOWLEDGEMENT. The author wishes to thank the referee for the idea to use White's result in [16] to simplify the proof of Theorem 5.21.

\section{REFERENCES}

1. Craw, I. G., Young, N. J., Regularity of multiplication in weighted group and semigroup algebras, Quart. J. Math. Oxford Ser. (2) 25 (1974), 351-358.

2. Dales, H. G., Banach Algebras and Automatic Continuity, Clarendon Press, Oxford, 2000.

3. Dales, H. G., Lau, A. T.-M., The second dual of Beurling algebras, Mem. Amer. Math. Soc. 177 (2005).

4. Dales, H. G., Polyakov, M. E., Homological properties of modules over group algebras, Proc. London Math. Soc. 89 (2004), 390-426.

5. Grønbæk, N., Amenability of discrete convolution algebras, the commutative case, Pacific J. Math. 143 (1990), 243-249.

6. Grønbæk, N., Amenability of weighted discrete convolution algebras on cancellative semigroups, Proc. Roy. Soc. Edinburgh Sect. A 110 (1988), 351-360.

7. Helemskii, A. Ya., Banach and Locally Convex Algberas, Oxford Science Publications, New York, 1993.

8. Helemskii, A. Ya., Homological essence of amenability in the sense of A. Connes: the injectivity of the predual bimodule, Math. USSR-Sb. 68 (1991), 555-566.

9. Helemskii, A. Ya., Some aspects of topological homology since 1995: a survey, 'Banach algebras and their applications' in Contemp. Math. 363 (2004), 145-179.

10. Runde, V., Amenability for dual Banach algebras, Studia Math. 148 (2001), 47-66.

11. Runde, V., Connes-amenability and normal, virtual diagonals for measure algebras. I., J. London Math. Soc. 67 (2003), 643-656.

12. Runde, V., Connes-amenability and normal, virtual diagonals for measure algebras, II, Bull. Austral. Math. Soc. 68 (2003), 325-328.

13. Runde, V., Dual Banach algebras: Connes-amenability, normal, virtual diagonals, and injectivity of the predual bimodule, Math. Scand. 95 (2004), 124-144.

14. Runde, V., Lectures on Amenability, Springer-Verlag, Berlin, 2002.

15. Tabaldyev, S. B., Noninjectivity of the predual bimodule of the measure algebra of infinite discrete groups, Math. Notes 73 (2003), 690-696. 
16. White, M. C., Characters on weighted amenable groups, Bull. London Math. Soc. 23 (1991), 375-380.

ST. JOHN'S COLLEGE

OXFORD

OX1 3JP UNITED KINGDOM

E-mail: matt.daws@cantab.net 